\title{
Impact of the food grade heat-killed probiotic and postbiotic oral lozenges in oral hygiene
}

\author{
Chiao-Wen Lin ${ }^{1,2}$, Yi-Tzu Chen ${ }^{1,2,3}$, Hsieh-Hsun Ho ${ }^{4}$, Yi-Wei Kuo ${ }^{4}$, Wen-Yang Lin ${ }^{4}$, Jui-Fen Chen ${ }^{4}$, \\ Jia-Hung Lin ${ }^{4}$, Cheng-Ruei Liu ${ }^{4}$, Chi-Huei Lin ${ }^{4}$, Yao-Tsung Yeh ${ }^{5}$, Ching-Wei Chen ${ }^{4}$, Yu-Fen Huang ${ }^{4}$, \\ Chen-Hung Hsu', Pei-Shan Hsieh ${ }^{4}$, Shun-Fa Yang ${ }^{6,7}$
}

${ }^{1}$ Institute of Oral Sciences, Chung Shan Medical University, Taichung, Taiwan
${ }^{2}$ Department of Dentistry, Chung Shan Medical University Hospital, Taichung, Taiwan
${ }^{3}$ School of Dentistry, Chung Shan Medical University, Taichung, Taiwan
${ }^{4}$ Research and Development Department, Bioflag Biotech Co., Ltd., Tainan, Taiwan
${ }^{5}$ Aging and Disease Prevention Research Center, Fooyin University, Kaohsiung, Taiwan
${ }^{6}$ Institute of Medicine, Chung Shan Medical University, Taichung, Taiwan
${ }^{7}$ Department of Medical Research, Chung Shan Medical University Hospital, Taichung, Taiwan

Correspondence to: Shun-Fa Yang; email: ysf@csmu.edu.tw

Keywords: oral cavity, probiotics, oral diseases, oral microbiota, immunity

Received: November 10, 2021 Accepted: February 22, 2022 Published: March 2, 2022

Copyright: (C) 2022 Lin et al. This is an open access article distributed under the terms of the Creative Commons Attribution License (CC BY 3.0), which permits unrestricted use, distribution, and reproduction in any medium, provided the original author and source are credited.

\section{ABSTRACT}

The oral cavity plays a crucial role in food digestion and immune protection. Thus, maintaining oral health is necessary. Postbiotic and heat-killed probiotic cells have shown increased antibacterial potential with stable viability compared with live strains. However, clinical evidence regarding their effect on oral health is insufficient. Therefore, in this study, we tested postbiotic lozenges of Lactobacillus salivarius subsp. salicinius AP-32, L. paracasei ET-66, and L. plantarum LPL28 and heat-killed probiotic lozenges of L. salivarius subsp. salicinius AP-32 and L. paracasei ET-66 for their effect on oral health. In total, 75 healthy individuals were blindly and randomly divided into placebo, postbiotic lozenge, and heat-killed probiotic lozenge groups and were administered the respective lozenge type for 4 weeks. Postbiotic and heat-killed probiotic lozenge groups demonstrated antibacterial activities with a considerable increase in $L$. salivarius in their oral cavity. Furthermore, their salivary immunoglobulin A, Lactobacillus, and Bifidobacterium increased. Subjective questionnaires completed by the participants indicated that participants in both the experimental groups developed better oral health and intestinal conditions than those in the placebo group. Overall, our study revealed that a food additive in the form of an oral postbiotic or heat-killed probiotic lozenge may effectively enhance oral immunity, inhibit the growth of oral pathogens, and increase the numbers of beneficial oral microbiota.

\section{INTRODUCTION}

The mucosal immune system of the oropharyngeal cavity, which is the opening gate for digestive and respiratory systems, plays a crucial role in preventing pathogen entry into the human body [1]. The oral cavity is a suitable environment for microbial colonization; 392 taxa with approximately 700 species and 1500 microbial genomes have been identified as residing therein. Firmicutes, Actinobacteria, Proteobacteria, Fusobacteria, Bacteroidetes, and Spirochaetes constitute $96 \%$ of all oral bacterial phyla $[2,3]$. Additionally, 85 fungal genera are found in the oral cavity [4]. Oral microbes colonize the oral 
cavity as a biofilm, which regulates oral homeostasis, oral immunity, food digestion, detoxification, inflammatory processes and is involved in disease prevention $[5,6]$.

A healthy oral environment consists of multiple symbiotic microbiota. Dewhirst et al. demonstrated major oral microbial phylum were Firmicutes (36.7\%), Bacteroidetes (17.3\%), Proteobacteria (17.1\%), Actinobacteria (11.6\%), Spirochaetes (7.9\%), Fusobacteria (5.2\%) [7]. However, poor oral hygiene may cause oral microbiota dysbiosis, which leads to dental bacterial plaque, gingivitis, and periodontitis [8]. In periodontitis, an outgrowth of pathogenic bacteria occurs, among which Actinobacillus actinomycetemcomitans, Porphyromonas gingivalis, and Fusobacterium nucleatum have been reported to be highly related to periodontal disease pathogenesis $[9,10]$. The World Health Organization (WHO) estimated that approximately $20 \%-50 \%$ of the global population has periodontitis [11]. Patients with periodontitis are at high risk of developing stroke, peripheral artery disease, and coronary heart disease [12]. The WHO suggested that strategies for periodontitis prevention include practicing oral hygiene, having a healthy diet, using fluoride and antimicrobial agents, and smoking cessation [13]. Studies have shown that live probiotic strains inhibit oral pathogens and are the rationale for periodontal treatment; they are similar to antibiotics but without the major concern of antimicrobial resistance [14]. However, manufacturing viable probiotics is not feasible owing to challenges regarding preservation and viability stabilization [15]. Metabolites of viable probiotic strains (postbiotic) such as 10-Hydroxy-cis-12-octadecenoic acid and heat-inactivated probiotics have shown the potential to alleviate the disruption of the gingival epithelial barrier caused by periodontitis [16].

In 2019, the panel of International Scientific Association for Probiotics and Prebiotics (ISAPP) defined the term 'postbiotics' as a preparation of inanimate microorganisms and/or their components that confers a health benefit on the host [17]. Postbiotics are often considered as metabolites secreted by probiotic strains during fermentation and consist of microbial cell fractions, polypeptides, peptidoglycan-derived muropeptides, bacteriocins, peroxides, pili-type structures, short-chain fatty acids, teichoic acid, folate, vitamins, lactic acid, and extracellular polysaccharides. Probiotic components benefit human health, provide nutritional support, competitively inhibit pathogenic bacteria, and regulate the immune system $[18,19]$. In addition, the fermentation products of lactic acid bacteria (postbiotics) have a unique flavor and beneficial nutrients and are therefore widely used in the food industry [20]. An in vitro study demonstrated that Lactobacilli postbiotics reduce colonization levels of A. actinomycetemcomitans, which are related to periodontitis [21]. However, clinical evidence proving that postbiotics reduce oral pathogenic bacteria and improve oral health is lacking. Additionally, our previous study revealed that certain heat-killed probiotics, including L. salivarius subsp. salicinius AP32 and L. paracasei ET-66, effectively limit the growth of oral pathogenic bacteria in vitro [22]. Current clinical data indicate that oral lozenges made of viable strains, including L. salivarius subsp. salicinius AP-32, L. paracasei ET-66, and L. plantarum LPL28, can increase beneficial microbiota in the oral cavity, reduce the colonization of periodontitis-related bacteria, and increase the levels of salivary immunoglobulin A (IgA) [23].

Based on previous in-vitro screening of viable probiotic strains for improving oral health, we further investigated whether heat-killed probiotic (ET-66 and AP-32), and postbiotic lozenges (LPL28, ET-66, and AP-32) modulate oral microbiota, inhibit oral infectious pathogens, and change salivary IgA levels $[22,23]$. The results can be applied in the production of supplementary foods for clinical oral health care in future.

\section{RESULTS}

Postbiotics of AP-32, ET-66, and LPL28 strains showed effective bactericidal effects on oral pathogens $S$. mutans, $P$. gingivalis, $F$. nucleatum subsp. polymorphum, and A. actinomycetemcomitans

The experimental design was revealed in supplementary data (Supplementary Figure 1). First, we generated the fermentation products of AP-32, ET66, and LPL28 as postbiotic oral lozenge, and examined its antipathogenic activity against oral pathogens (Figure 1). Compared with the postbiotic of a commercially available strain (LGG), the fermentation products (postbiotics) of AP-32, ET-66, and LPL28 strains had stronger bactericidal effects on oral pathogens, particularly $S$. mutans and $A$. actinomycetemcomitans (Figure 1A). The inhibition rates of $S$. mutans were significantly higher with the use of the postbiotics of AP-32 (62.6\%), ET66 (99.86\%, p < 0.001), and LPL28 (94.35\%, $\mathrm{p}<0.001)$ than with the use of LGG postbiotic (41.64\%). In addition, the inhibition rates of $A$. actinomycetemcomitans were significantly higher with the use of the postbiotics of AP-32 $(17.92 \%, \mathrm{p}<0.05)$, ET-66 $(77.86 \%, \mathrm{p}<0.001)$, and LPL28 $(24.28 \%, \mathrm{p}<$ $0.001)$ than with the use of LGG postbiotic $(13.69 \%)$. All postbiotics effectively inhibited periodontal pathogens $P$. gingivalis BCRC 17689, $P$. gingivalis BCRC 17688, and F. nucleatum subsp. polymorphum 


\section{In vitro anti-oral pathogenic activity test for individual postbiotic}

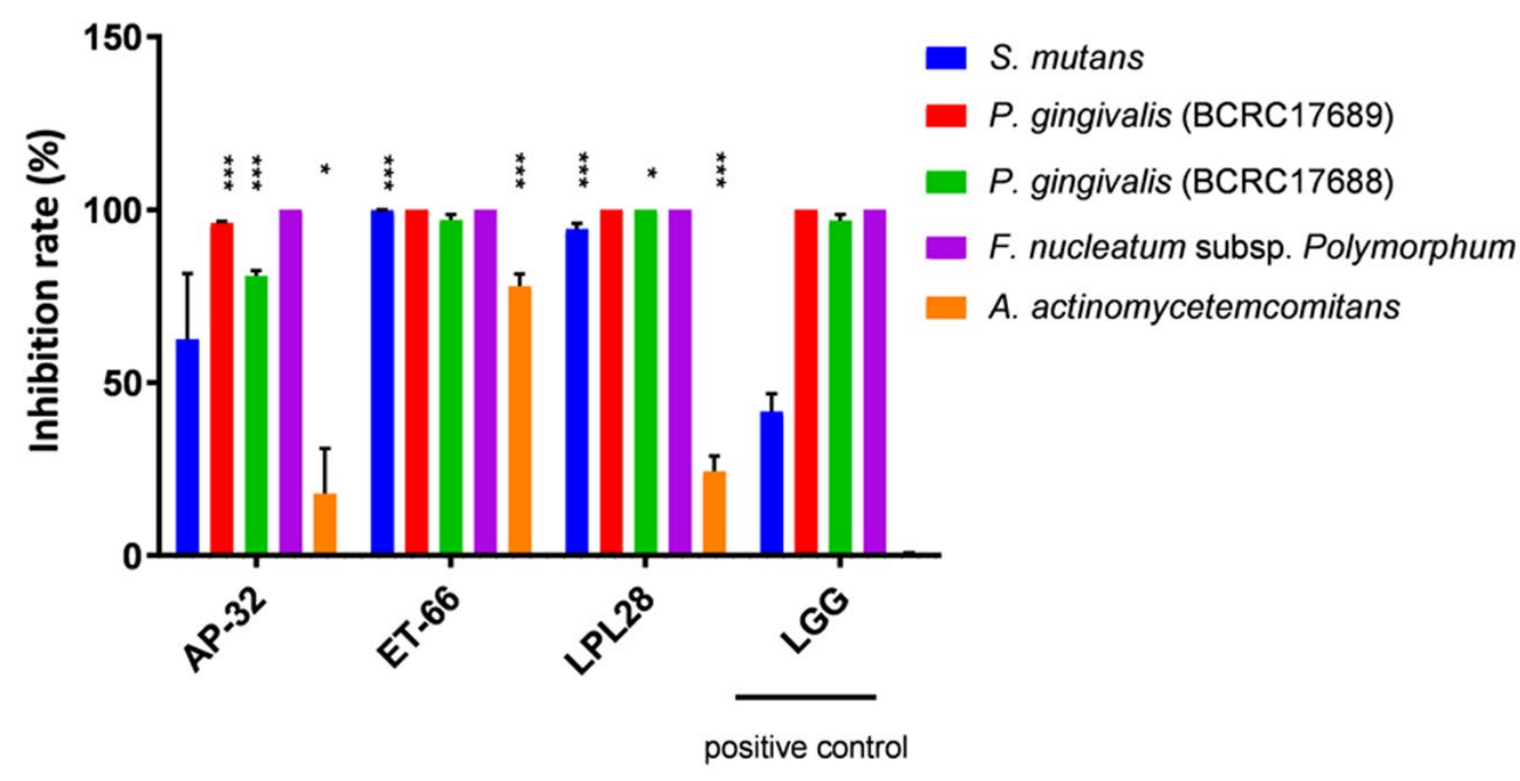

B

In vitro anti-oral pathogenic activity test for oral lozenges

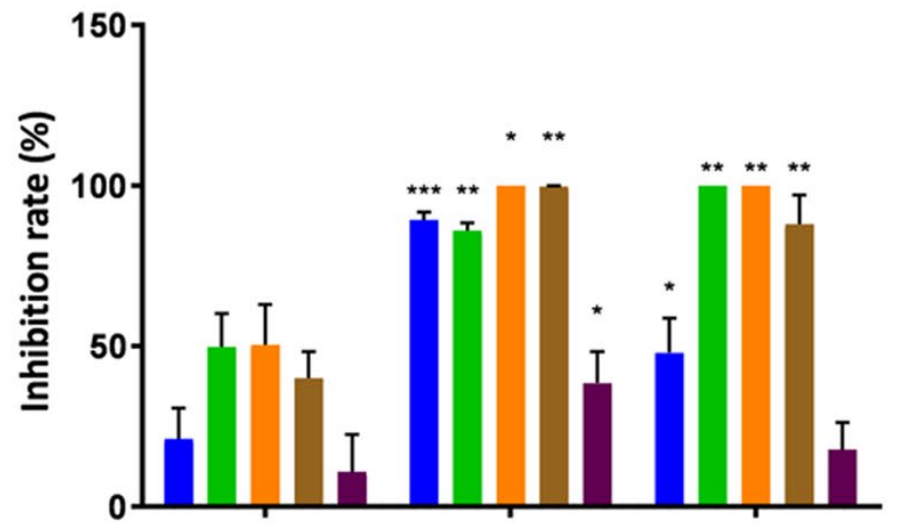

- S. mutans

- P. gingivalis (BCRC17689)

- P. gingivalis (BCRC17688)

- F. nucleatum subsp. Polymorphum

- A. actinomycetemcomitans

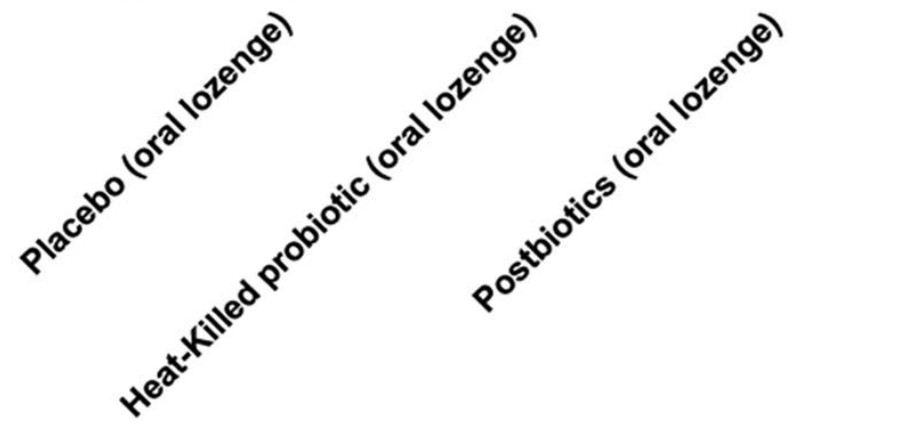

Figure 1. In vitro test for determining the antipathogenic activity of (A) individual postbiotic and (B) probiotic oral lozenges against oral pathogens. (A) Postbiotics of AP-32, ET-66, and LPL28 showed strong antibacterial activities compared with the positive control of LGG postbiotic. (B) Heat-killed AP-32 and ET-66 were used as inactivated probiotics, whereas metabolites of AP-32, ET-66, and LPL28 were used as postbiotics. ${ }^{*} p<0.05, * * p<0.01$, and $* * * p<0.001$ compared with the positive control group (LGG postbiotic) or the placebo group (without the postbiotic). Data are presented as mean \pm SD. 
(Figure 1A). LPL28 postbiotics had a higher inhibition rate of $P$. gingivalis BCRC $17688\left(100 \%, \mathrm{p}<0.05^{*}\right)$ than did LGG postbiotic (96.83\%).

\section{Postbiotic and heat-killed probiotic lozenges were effective, demonstrating in vitro bactericidal ability against oral pathogens}

We prepared two oral lozenges, one with the postbiotics of AP-32, ET-66, and LPL28 and the other with heatkilled AP-32 and ET-66 probiotics for an in vitro bactericidal test before launching clinical trials (Figure 1B). The postbiotic oral lozenge group showed a significant increase in the inhibition rates of $S$. mutans, $P$. gingivalis (BCRC 17689), P. gingivalis (BCRC 17688), $F$. nucleatum, and A. actinomycetemcomitans, namely increases of $47.97 \%(\mathrm{p}<0.05$, placebo $=21.02 \%), 100 \%$ $(\mathrm{p}<0.01$, placebo $=49.74 \%), 100 \%(\mathrm{p}<0.01$, placebo $=$ $50.38 \%$ ), and $87.88 \%(\mathrm{p}<0.01$, placebo $=39.96 \%)$, respectively, compared with the placebo group. The inhibition rate of $A$. actinomycetemcomitans in the experimental group slightly increased without a significant difference compared with the placebo group (oral lozenge: 17.78\%; placebo: 10.85\%). The heat-killed probiotic group significantly inhibited $S$. mutans, $P$. gingivalis (BCRC 17689), P. gingivalis (BCRC 17688), $F$. nucleatum, and $A$. actinomycetemcomitans by $89.32 \%$ $(\mathrm{p}<0.001), 85.91 \%(\mathrm{p}<0.01), 95.32 \%(\mathrm{p}<0.05)$, $91.75 \%(\mathrm{p}<0.01)$, and $48.46 \%(\mathrm{p}<0.05)$, respectively.

\section{Postbiotic and heat-killed probiotic lozenges effectively reduced pathogenic colonies in the saliva samples of participants}

The 75 selected participants were randomly assigned to three groups: placebo, postbiotic lozenge, and heat-killed probiotic lozenge. We collected saliva samples at weeks 0,2 , and 4 after oral lozenge intake initiation and measured changes in their microbiota. Plaque weight was $0.37 \pm 0.16 \mathrm{~g}$ at week 0 , and the initial $S$. mutans in saliva $(\mathrm{CFUs} / \mathrm{mL})$ was $4.25 \mathrm{E}+06 \pm 2.90 \mathrm{E}+06$. The postbiotic lozenge significantly reduced the oral $S$. mutans bioburden to $60 \%$ (median) at week 4 (compared with the postbiotic lozenge at week 0 and placebo at week 4; $\mathrm{p}<0.05$ for both), and the heat-killed probiotic lozenge significantly reduced $S$. mutans to $61 \%$ (median) at week 4 (compared with the probiotic lozenge at week 4, $\mathrm{p}<0.05$; Figure 2A). Plate Count Agar (PCA) is a common microbiological growth medium used to monitor total viable bacterial populations of a sample [24]. The PCA agar plate was used to analyze the total bacterial population in the oral cavity. The result indicated that administrating postbiotic lozenges significantly decreased the total bacterial load to $98 \%$ at week 2 (compared with placebo at week 2, 160\%, p < 0.05; Figure 2B) and to $104 \%$ at week 4 (compared with placebo at week $4[187 \%]$ and postbiotic lozenge at week $0 ; \mathrm{p}<0.05$ for both).

\section{Postbiotic and heat-killed probiotic lozenges effectively increased beneficial microbial strains in saliva samples}

The change in the Bifidobacterium population in the participants' oral cavity was further measured after oral lozenge intake. The results revealed that the postbiotic oral lozenge significantly increased the Bifidobacterium population to $141 \%$ at week 4 (compared with the postbiotic lozenge at week 0 and placebo at week 4 [64\%], $p<0.05$ for both; Figure 2C). Furthermore, the heat-killed probiotic lozenge significantly increased the salivary Bifidobacterium population to $111 \%$ at week 2 (compared with the probiotic lozenge at week 0 and placebo at week $4, \mathrm{p}<0.05$ for both) and to $114 \%$ at week 4 (compared with the probiotic lozenge at week 0 $[\mathrm{p}<0.05]$ and placebo at week $4[\mathrm{p}<0.01])$.

The measurement of the Lactobacillus population in saliva samples revealed that the postbiotic oral lozenge significantly increased the Lactobacillus population to $135 \%$ at week 2 (compared with the postbiotic oral lozenge at week $0[\mathrm{p}<0.05]$ and placebo at week $2[\mathrm{p}<$ 0.01 ]; Figure 2D) and to $227 \%$ at week 4 (compared with the postbiotic oral lozenge at week 0 and placebo at week $4 ; \mathrm{p}<0.001$ for both). Furthermore, the heat-killed probiotic lozenge significantly increased the salivary Lactobacillus population to $123 \%$ at week 2 (compared with the heat-killed probiotic lozenge at week 0 and placebo at week $4 ; \mathrm{p}<0.05$ for both) and to $201 \%$ at week 4 (compared with the heat-killed probiotic lozenge at week 0 and placebo at week $4 ; \mathrm{p}<0.001$ for both).

\section{Postbiotic and heat-killed probiotic lozenges effectively increased $\operatorname{IgA}$ concentration in saliva samples}

IgA concentration in saliva increased significantly after consuming postbiotic oral lozenges (Figure 3). The postbiotic lozenge significantly increased saliva IgA to $126 \%$ at week 2 (compared with the postbiotic lozenge at week $0[\mathrm{p}<0.05]$ and placebo at week $2[\mathrm{p}$ $<0.01]$ ) and to $168 \%$ at week 4 (compared with the postbiotic lozenge at week 0 and placebo at week $4 ; p$ $<0.001$ for both). Moreover, the heat-killed probiotic lozenges significantly increased salivary IgA to $122 \%$ at week 2 (compared with the probiotic lozenges at week 0 and placebo at week $2 ; \mathrm{p}<0.01$ for both) and to $163 \%$ at week 4 (compared with the probiotic lozenge at week 0 and placebo at week 4; p $<0.001$ for both). However, plaque weights did not change much with oral lozenge intake for 4 weeks (Supplementary Figure 2). 
A

S. mutans in saliva

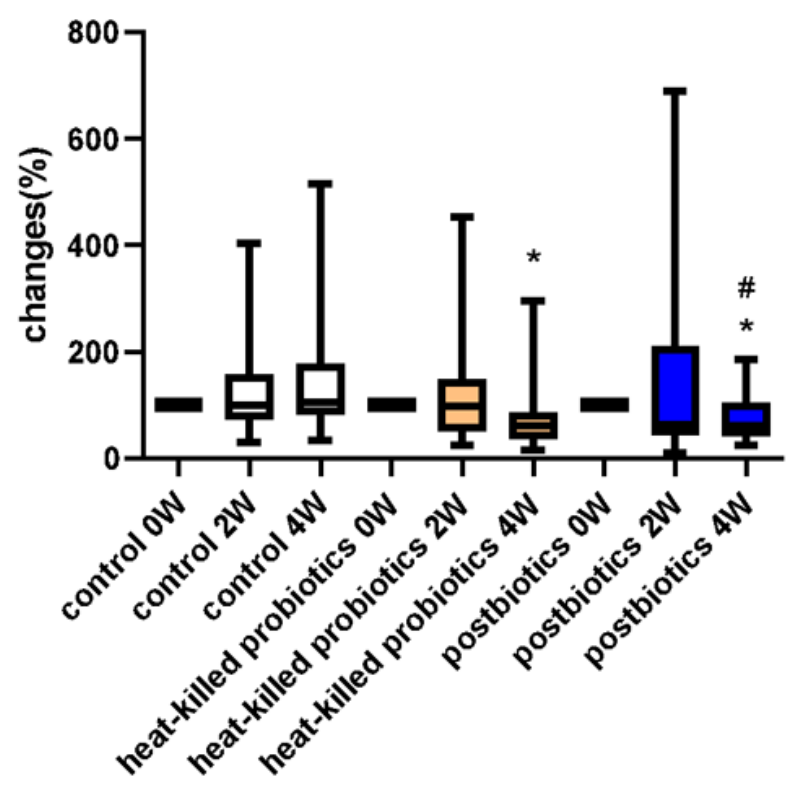

C Bifidobacterium population in saliva

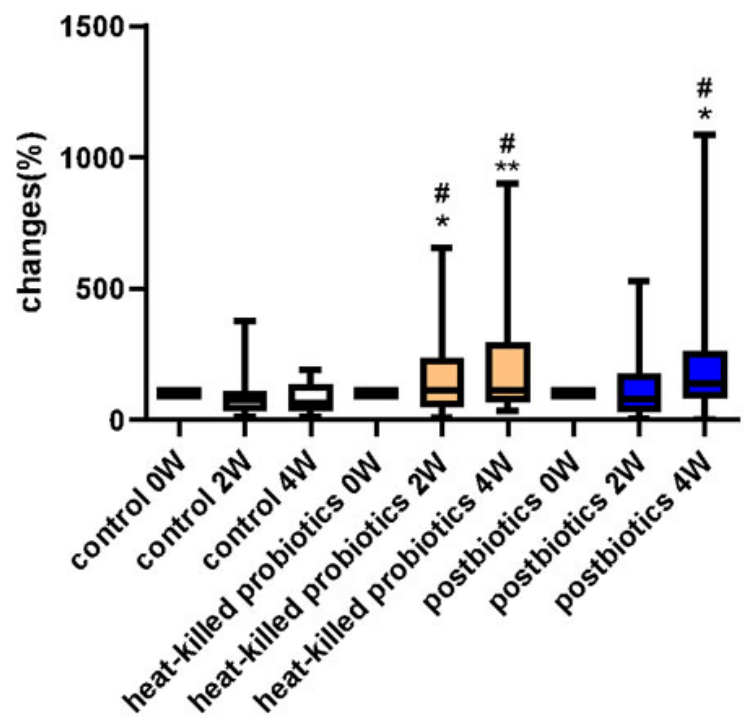

B

Total salivary aerobic bacteria in PCA
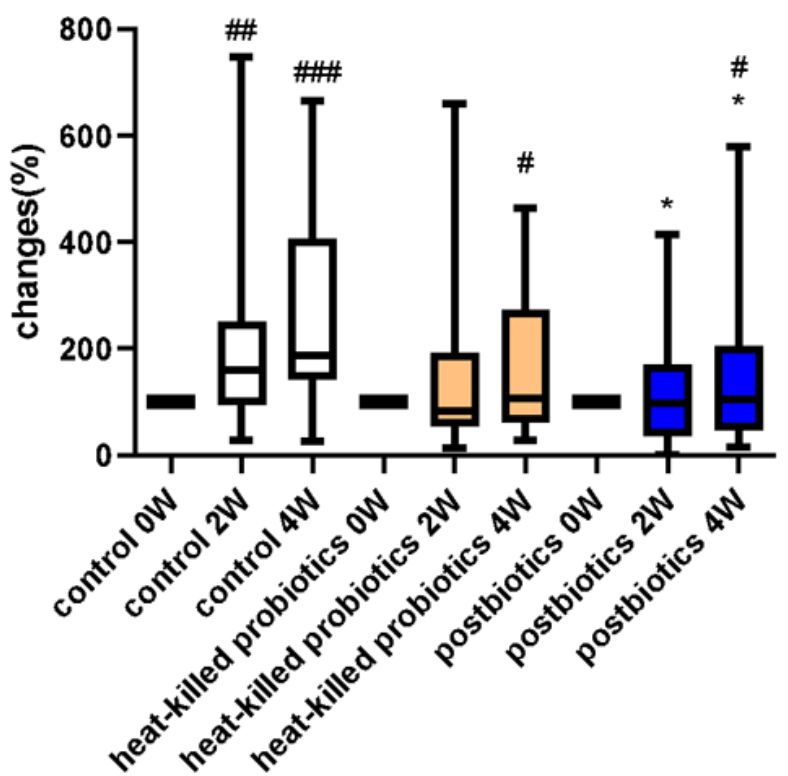

D Lactobacillus population in saliva
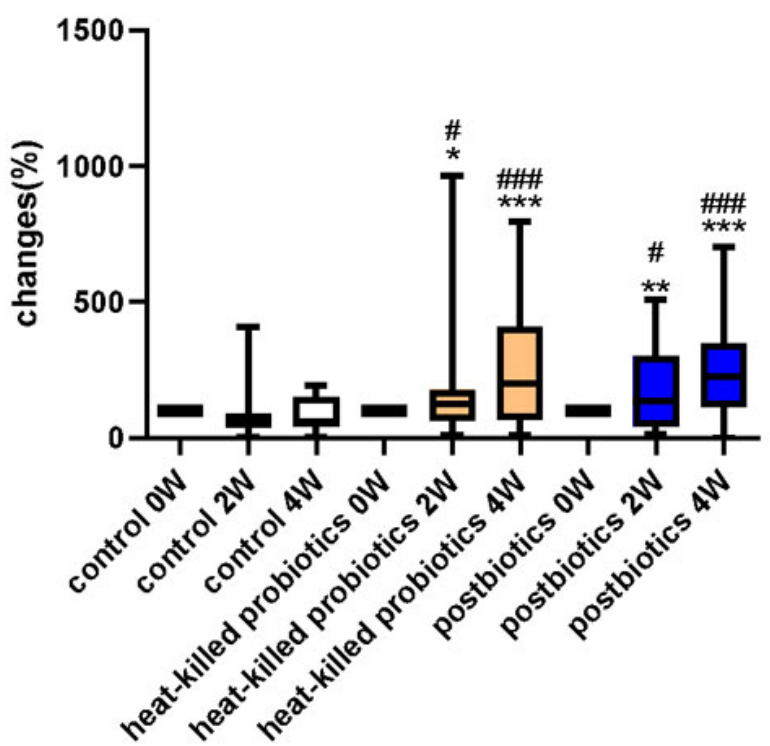

Figure 2. Microbial change (\%) in saliva samples. Change (\%) in the population of (A) S. mutans, (B) total bacteria, (C) Bifidobacterium, and (D) Lactobacillus in participants' saliva at 0,2 , and 4 weeks of oral lozenge intake. The oral lozenges contained postbiotics or heat-killed cells. Participants in the control group consumed placebo lozenges without the postbiotic content $(* p<0.05, * * p<0.01$, and $* * * p<0.001$ compared with the control group; \#p $<0.05$, \#\#p $<0.01$, and \#\#\# < 0.001 in reference to the values at week 0$)$. Data are presented as medians ( $n=25$ in each group). 
NGS detected oral microbiota changes with oral lozenge intake

We used the NGS technique to analyze microbiota changes in saliva samples with oral lozenge intake. Species heatmap (\%) demonstrated that L. salivarius significantly increased to $0.03 \%$ (compared with the placebo group, $\mathrm{p}<0.05)$ at 4 weeks after consuming postbiotic oral lozenges. Additionally, heat-killed probiotic lozenges significantly increased $L$. salivarius to $0.06 \%$ (compared with the placebo group, $\mathrm{p}<0.05$; Supplementary Figure 3). The result confirmed our previous findings on plate culturing for quantifying Lactobacillus in saliva samples (Figure 2D).

LEfSe analysis was used to identify the oral microbiota change between before and after oral lozenge intake. Nine differential bacterial taxa significantly increased after the intake of heat-killed probiotic lozenges, including Lactobacillus (Figure 4A). In total, 10 oral bacterial clades significantly increased with the intake of postbiotic lozenges, including Lactobacillus (Figure 4B).

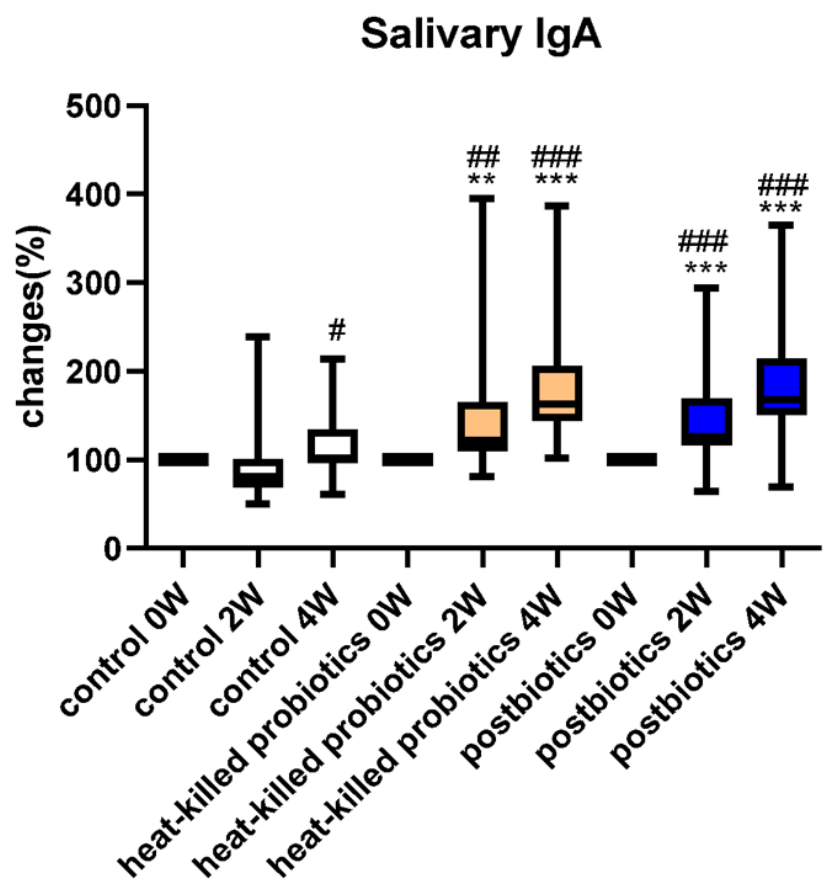

Figure 3. Oral lozenges significantly increased salivary IgA levels. Change in Lactobacillus (\%) in participants' saliva at 0, 2, and 4 weeks with the intake of oral lozenges. Oral lozenges contained postbiotics or heat-killed cells. Participants in the control group consumed placebo lozenges without the postbiotic content $(* p<0.05, * * p<0.01$, and $* * * p<0.001$ compared with the control group; \#p < 0.05, \#\#p < 0.01, and \#\#\# < 0.001 in reference to values at week 0 ). Data are presented as medians ( $n$ $=25$ in each group).
We further analyzed statistical alteration in certain oral bacterial strains with the intake of heat-killed probiotic or postbiotic lozenges (Figure 5A-5F). Pathogenic Veillonella spp. $(\mathrm{p}<0.01)$, Actinomyces graevenitzii F0530 ( $<<0.05)$, and Prevotella sp. C561 ( $<00.001)$ significantly decreased by 4 weeks of treatment with heat-killed probiotic lozenges. However, the postbiotic oral lozenges significantly reduced the growth of Selenomonas 3 spp. $(\mathrm{p}<0.01)$ and Prevotella sp. oral clone FW035 $(\mathrm{p}<0.01)$. L. salivarius significantly increased with the intake of heat-killed probiotic $(\mathrm{p}<$ $0.01)$ and postbiotic $(\mathrm{p}<0.001)$ lozenges.

Oral lozenges relieved the symptoms of mouth sores, constipation, and gastroesophageal reflux based on health questionnaire analysis

The severity scores for mouth sores or pustule formation decreased to $0.04 \pm 0.2(\mathrm{p}<0.01$, compared with the placebo group) and $0.08 \pm 0.28$ ( $p<0.05$, compared with the placebo group) after postbiotic lozenge administration for 2 and 4 weeks, respectively (Supplementary Table 1). Furthermore, the heat-killed lozenges relieved the symptoms of mouth sores at weeks $2(0.23 \pm 0.51, \mathrm{p}<0.05$, compared with the placebo group $)$ and $4(0.15 \pm 0.37, \mathrm{p}<0.05$, compared with the placebo group), respectively. The scores for constipation declined to $0.12 \pm 0.33(\mathrm{p}<0.01)$ after 4 weeks of postbiotic lozenge intake (Supplementary Table 2). Additionally, 4 weeks after postbiotic oral lozenge intake, the symptoms of gastroesophageal reflux, cold, and drowsiness significantly decreased to $0.12 \pm 0.33(\mathrm{p}<0.05), 0.16 \pm 0.37(\mathrm{p}<0.05)$, and 0.12 \pm 0.33 ( $\mathrm{p}<0.01)$, respectively. Moreover, the heatkilled probiotic lozenges diminished the symptoms of constipation, gastroesophageal reflux, cold, and drowsiness at week 4 to $0.15 \pm 0.46(\mathrm{p}<0.05), 0.19 \pm$ $0.4(\mathrm{p}<0.05), 0.12 \pm 0.43(\mathrm{p}<0.05)$, and $0.08 \pm 0.27(\mathrm{p}$ $<0.01)$, respectively.

\section{DISCUSSION}

Based on previous research of viable strain-specific approach [25], we selected three of most appropriate strains to generate oral health promoting products of heat-killed probiotic (ET-66 and AP-32), and postbiotic lozenges (LPL28, ET-66, and AP-32) [22, 23]. Several studies have tested the effect of certain live probiotic strains on oral health. For example, probiotic Streptococcus salivarius was reported to reduce severe oral halitosis [26]. However, no study has investigated the role of the postbiotics on regulating oral microbiota and oral immunity. At the beginning of this research, we used in vitro antipathogenic assay to demonstrate that individual postbiotics of AP-32, ET-66, and LPL28 can limit the growth rate of oral pathogenic 
Heat_Killed-After

Heat_Killed-Before

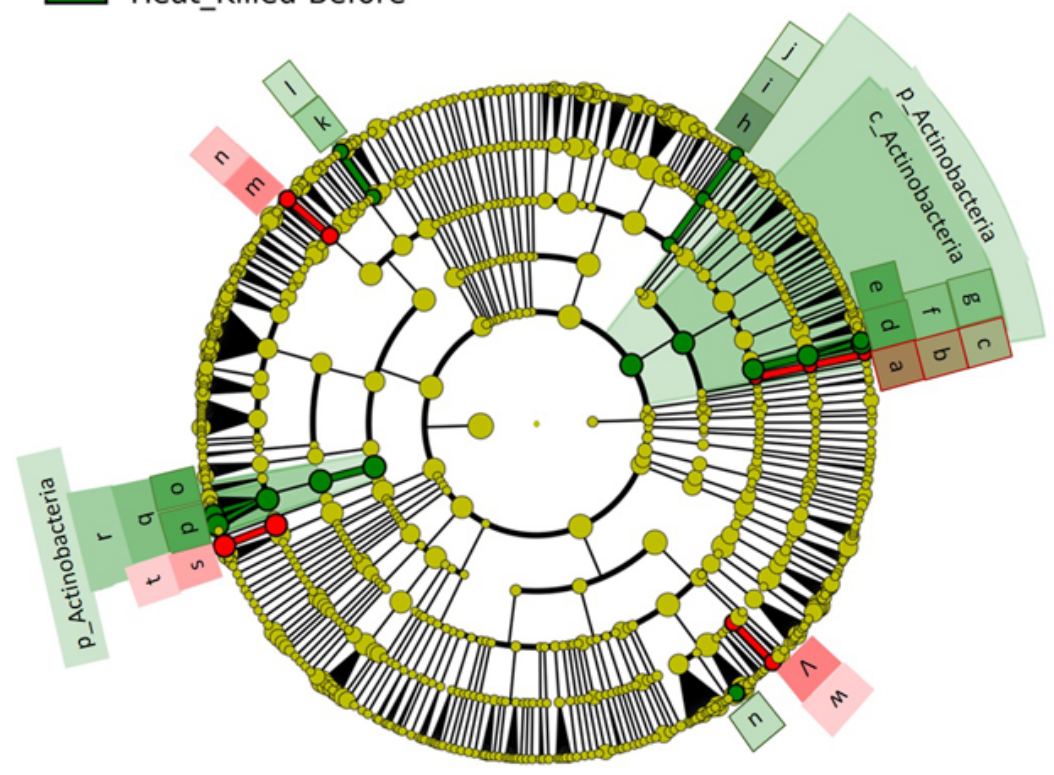

B

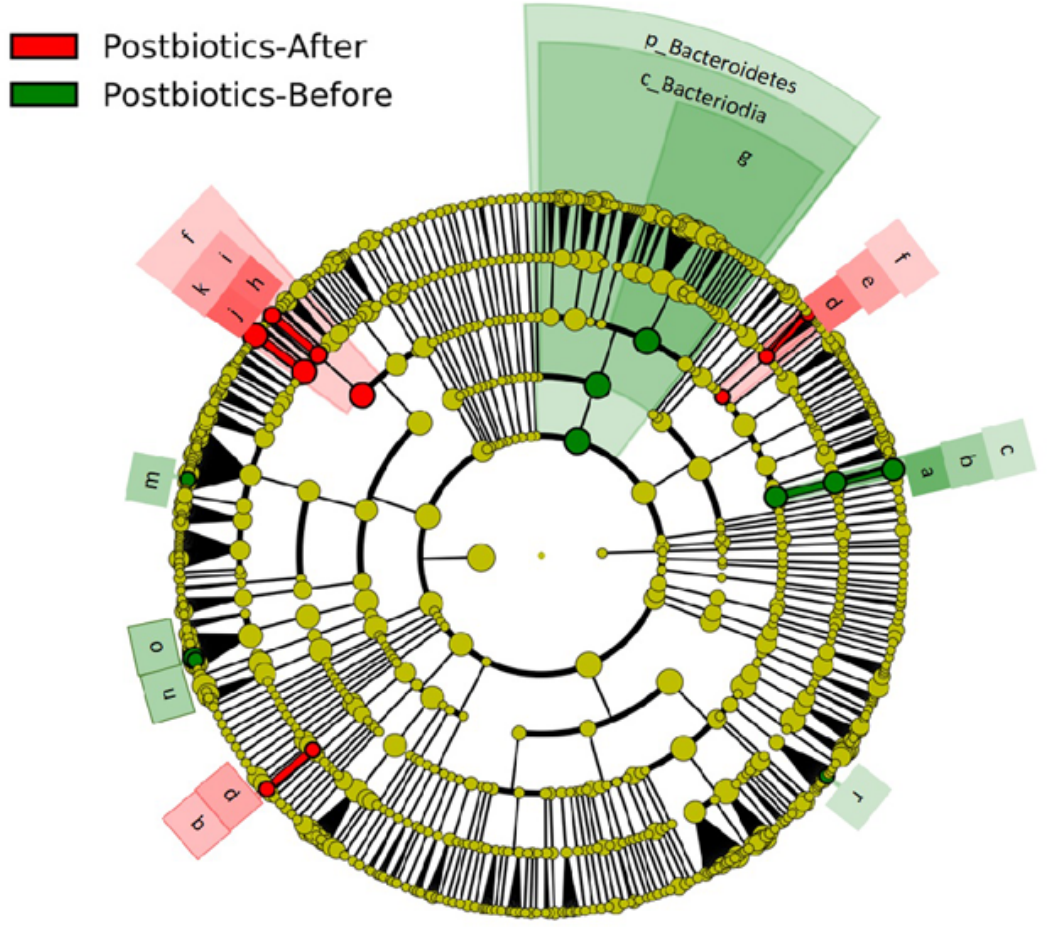

a: c_Actinobacteria_unclassified

b: c_Actinobacteria_unclassified

c: c_Actinobacteria_unclassified

d: g_Actinomyces

e: g_F0332

f: f_Actinomycetaceae

g: o_Actinomycetales

$\square$ h: c_Bacteroidia_unclassified

i: c_Bacteroidia_unclassified

$\square$ j: c_Bacteroidia_unclassified

k: g_Exiguobacterium

I: f_FamilyXII

m: g_Lactobacillus

n: f_Lactobacillaceae

o: g__Megasphaera

$\square$ p: g_Veillonella

$\square$ q:f_Veillonellaceae

r: o_selenomonadales

s: g_fusobacterium

$\square \mathrm{t}: \mathrm{f}$ __Fusobacteriaceae

u: g_Alysiella

v: o_Betaproteobacteriales_unclassified

w: o_Betaproteobacteriales_unclassified

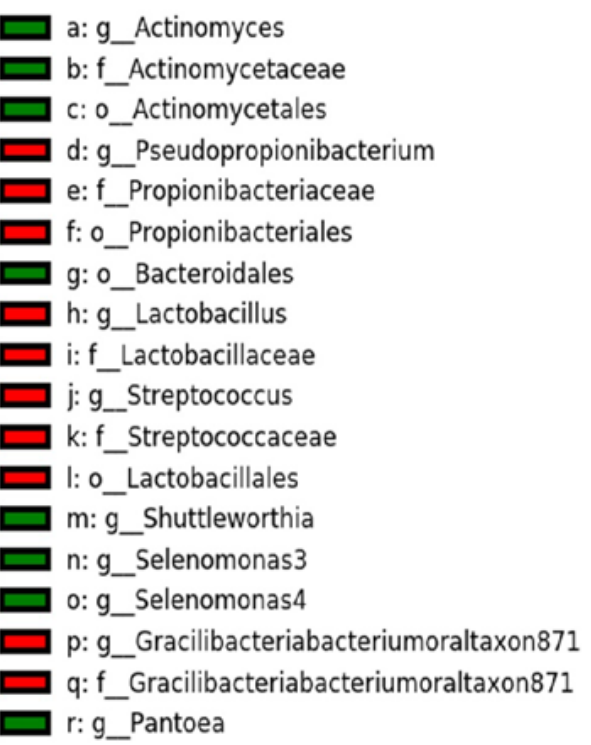

Figure 4. LEfSe analysis of differential oral microbiota before and after 4 weeks of consuming oral lozenge. Comparing changes in oral microbiota with the intake of $(\mathbf{A})$ heat-killed probiotic lozenges and $(\mathbf{B})$ postbiotic lozenges ( $n=25$ in each group). 
bacteria S.mutans and periodontal pathogens $P$. gingivalis BCRC 17689, $P$. gingivalis BCRC 17688, F. nucleatum subsp. polymorphum, and $A$. actinomycetemcomitans (Figure 1A). Furthermore, the postbiotic lozenge made from mixed metabolites of AP-32, ET-66, and LPL28 exhibited reliable antibacterial function in vitro (Figure 1B). The heatkilled probiotic lozenges showed better inhibition rate in $S$. mutans and A. actinomycetemcomitans than postbiotic lozenges. Moreover, the heat-killed probiotic lozenges presented excellent bactericidal ability in oral pathogens, which was in accordance with previous findings on individual heat-killed strains [22]. However, the oral lozenges (made from 3 mixed postbiotics) did not inhibit $S$. mutans and $A$. actinomycetemcomitans better than individual ET-66 postbiotic did. This may be because of a lower concentration of functional ingredients in the oral lozenge than in an individual postbiotic $(50 \mathrm{mg}$ individual postbiotic versus $50 \mathrm{mg}$ of mixed postbiotics/1 g of lozenge). Besides, previous study revealed viable probiotic lozenges had an excellent inhibition rate (nearly 100\%) in five oral pathogenic bacteria $S$. mutans and $P$. gingivalis BCRC 17689, $P$. gingivalis $\mathrm{BCRC} 17688, F$. nucleatum subsp. polymorphum, and A. actinomycetemcomitans [23]. Higher dosage of heat-killed and postbiotic lozenges are presumed to achieve similar pathogenic growth inhibition rate to viable probiotic lozenges. The half maximal inhibitory concentration (IC50) for heatkilled and postbiotic lozenges in limiting oral growth rate should be tested in future.

Next, we validated the antipathogenic ability of heatkilled probiotic or postbiotic lozenges through the detection of changes in the microbial number in saliva.
A

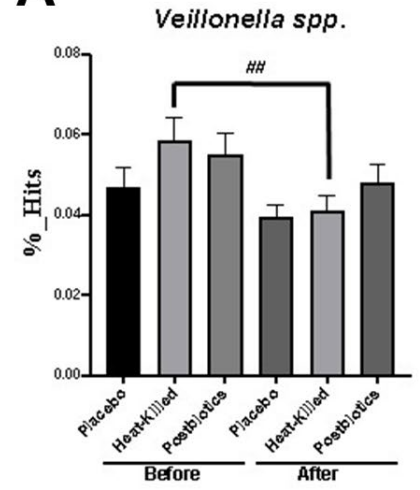

D

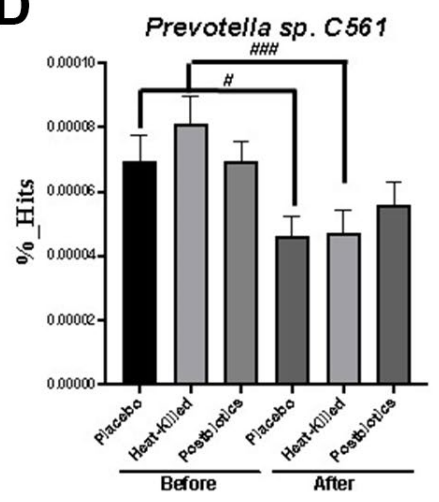

B

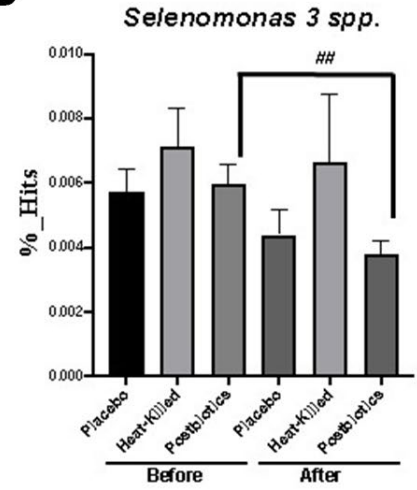

$\mathbf{E}$

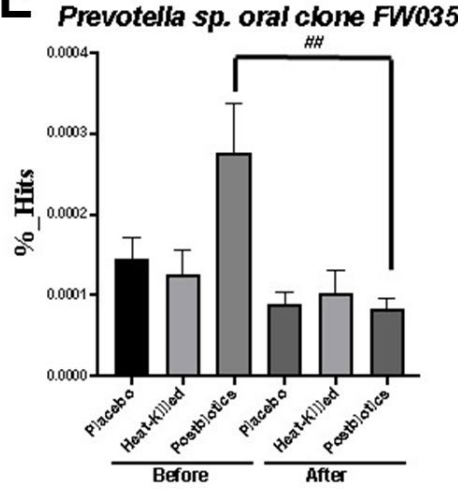

C

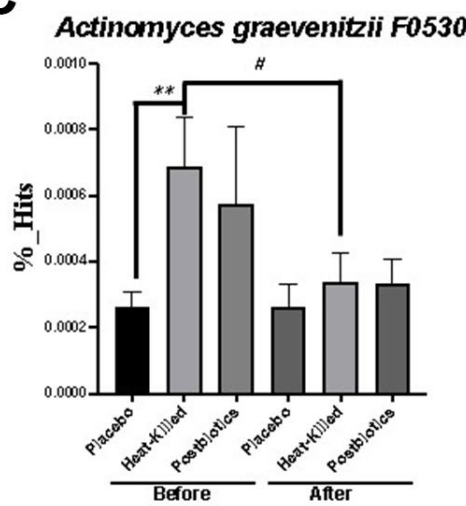

F

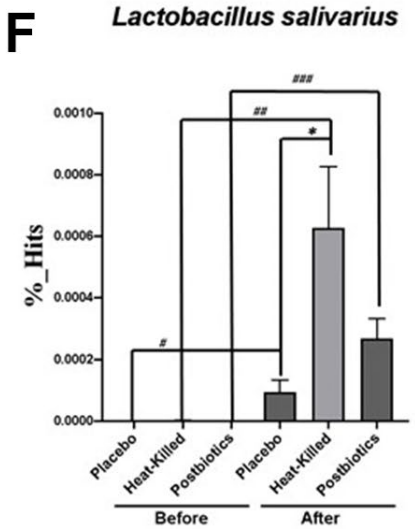

Figure 5. Significant changes in specific oral bacterial strains after consuming heat-killed probiotic or postbiotic oral lozenges. Changes in (A) Veillonella spp., (B) Selenomonas 3 spp., (C) Actinomyces graevenitzii F0530, (D) Prevotella sp. C561, (E) Prevotella sp. oral clone FW035, and (F) L. salivarius after consuming heat-killed probiotic or postbiotic oral lozenges were analyzed through the LEfSe analysis. Comparing changes in oral bacterial concentration (\%) in participants' saliva at 0 (before) or 4 weeks (after) of oral lozenge intake. The oral lozenges contained postbiotics or heat-killed cells. Participants in the control group consumed placebo lozenges without the postbiotic content $(* p<0.05, * * p<0.01$, and $* * * p<0.001$ compared with the control group; \#p $<0.05, \# \#<<0.01$, and \#\#\#p<0.001 in reference to the values at week 0 ). Two-tailed t-tests was performed to analyze the statistical difference of experimental results. Data are presented as means \pm SDs ( $n=25$ in each group). 
Compared with the placebo group, the postbiotic and heat-killed lozenge groups exhibited significantly reduced numbers of $S$. mutans and total bacteria at week 4 , but no significantly difference at week 2 (Figure 2A, 2B). $S$. mutans is the main pathogen involved in the initiation of dental caries and exhibited a positive correlation with periodontitis [27]. In addition, a high ratio of $S$. mutans DNA was discovered in cardiovascular specimens [28]. Thus, reducing oral $S$. mutans numbers with postbiotic lozenge intake may prevent dental cavity progression, periodontitis, and cardiovascular diseases. Moreover, Bifidobacterium and Lactobacillus in saliva (cultured on MRS agar plate) revealed that cell numbers increased with the intake of heat-killed probiotic or postbiotic lozenges (Figure 2C, 2D).

Furthermore, postbiotic or heat-killed probiotic lozenges increased IgA concentration in saliva (Figure 3). IgA constitutes $10 \%-20 \%$ of the serum immunoglobulin, second only to IgG. Moreover, IgA present in the mucosal tissues of the oral cavity, digestive tract, and respiratory tract prevents pathogen invasion. Additionally, IgA is present in saliva, tears, and breast milk, particularly in that with high colostrum. No IgA antibody is present in neonatal serum, but newborns obtain IgA secreted from breast milk [29]. Carbohydrate intake may reduce IgA concentration in saliva [30]. Furthermore, salivary $\operatorname{IgA}$ acts as the frontline mucosal immune defense against the entry of respiratory pathogens, including severe acute respiratory syndrome coronavirus 2 [31]. Therefore, novel postbiotic or heat-killed probiotic lozenges, which effectively increase salivary IgA concentration, improve bacteriostatic activities, and increase oral populations of beneficial bacteria, may be a potential food product in improving oral health and preventing further infection.

Additionally, we used LEfSe analysis to detect significant changes in oral microbiota after the administration of heat-killed probiotic or postbiotic lozenges (Figure 4). Both heat-killed and postbiotic lozenges showed the ability to significantly increase Lactobacillus in the oral cavity. Furthermore, heatmap results of NGS analysis showed an increase in $L$. salivarius level (Supplementary Figure 3). Thus, oral lozenges containing postbiotic or heat-killed probiotic cells promoted the growth and colonization of beneficial microorganism in the oral cavity. In addition, the upregulation of $L$. salivarius in oral microbiota has been reported to promote anticariogenic effects [32, 33]. Moreover, an animal study revealed that $L$. salivarius subsp. salicinius AP-32 can eradicate Helicobacter pylori infection in addition to improving oral health [34].

Based on previous findings that probiotic strains of AP-32, ET-66 and LPL28 effected oral microbiota
[23] and viable strains may improve salivary IgA via up-regulating anti-inflammatory cytokines, IL-10 and TGF-beta [35]. Besides, a previous animal study discovered the mixed viable probiotic strains of Lactobacillus salivarius subsp. salicinius AP-32, L. johnsonii MH-68, L. reuteri GL-104, and Bifidobacterium animalis subsp. lactis CP-9, significantly increased SCFA and MCFA levels. The elevated SCFA and MCFA levels may affect the populations of gut microbiota [36]. The secreted SCFA and MCFA from viable probiotic strains may affect the oral bacterial populations. Some metabolites such as butyrate may stimulate the formation of periodontal/periapical tissues at low or high concentrations [37-39].

However, the detailed mechanism of how three mixed viable probiotic strains (AP-32, ET-66, and LPL28) altered the oral microbiome should be tested in the future. Here, we further discovered that postbiotic would also improve oral microbiota. Nevertheless, the clinical oral health improving function should be tested after stopping consuming postbiotic lozenges. A larger scale clinical analysis of oral microbiota and metabolite profiling for the development of personalized oral therapy in the future [40].

The heat-killed lozenges were efficacious in reducing growth of pathogenic Veillonella spp., A. graevenitzii F0530, and Prevotella sp. C561. Moreover, the postbiotic lozenges reduced the growth of Selenomonas 3 spp. and Prevotella sp. oral clone FW035 (Figure 5). Veillonella spp. has been reported to be associated with halitosis [41] and dental caries [42]. The overgrowth of Prevotella spp. may lead to halitosis [41] and periodontal disease [43]. $A$. graevenitzii has been discovered to cause pulmonary abscess [44], pneumonia [45], and dental caries [42]. Thus, heat-killed probiotic lozenges might improve the oral smell and oral hygiene by reducing the oral population of Veillonella spp., Prevotella spp., and A. graevenitzii. Additionally, postbiotic oral lozenges may significantly reduce Selenomonas spp. and Prevotella spp., which are associated with halitosis [41]. Moreover, the oral health questionnaires presented that heat-killed and postbiotic lozenges would significantly improve symptoms of ruptured mouth, drool (Supplementary Table 1), constipation, gastroesophageal reflux, cold, drowsiness (Supplementary Table 2). The results of questionnaires at present study are in accordance with previous findings in viable probiotic lozenges. The questionnaires for viable probiotic lozenge present additional improvements in teeth bleeding, sore throat, and stomach pain [23]. 
Finally, Ishikawa, K. H. et al. demonstrated postbiotics would effectively limit the formation of biofilm formation and growth rate of $A$. actinomycetemcomitans [21]. At present study, it demonstrated that postbiotic significantly reduced the survival rate of other oral pathogens including $S$. mutans and periodontal pathogens $P$. gingivalis BCRC 17689, $P$. gingivalis BCRC 17688, F. nucleatum subsp. polymorphum. We also measured changes in participants' salivary IgA and oral microbiota by consuming lozenges of postbiotic AP-32 (L. salivarius subsp. salicinius), ET-66 ( $L$. paracasei), and LPL28 (L. plantarum). The different mixing proportion of three postbiotics effected on oral hygiene should be tested in future.

In conclusion, the postbiotics and heat-killed probiotics have the advantages of preservation and stable viability over viable strains. Here, we found that lozenges of postbiotic AP-32 (L. salivarius subsp. salicinius), ET-66 (L. paracasei), and LPL28 ( $L$. plantarum) and the heat-killed probiotics of AP-32 ( $L$. salivarius subsp. salicinius) and ET-66 (L. paracasei) were beneficial to oral health. Previous study demonstrated that three strains had excellent antimicrobial activity in zone of inhibition test. The present clinical study revealed that postbiotic or heatkilled probiotic lozenges could effectively reduce the number of $S$. mutans in the oral cavity, increase $L$. salivarius in oral microbial flora, increase salivary IgA concentration, and decrease oral infections. Furthermore, results from the subjective questionnaire revealed that improved oral health was associated with attenuated intestinal symptoms, relieved constipation, and reduced gastroesophageal reflux, stomach pain, colds, and sense of drowsiness. This study suggested that deactivated probiotic cells and their postbiotics can serve as supporters to optimize the efficacy of oral health supplements. However, further experiments are required.

According to the current manufacturing regulations of cosmetics and cleaning products in various countries, the inactive substances of functional lactic acid bacteria are more suitable for the industrial application. Therefore, this study presented potential food-grade supplementations for promoting oral health and applicable food industrial products in the future.

\section{MATERIALS AND METHODS}

\section{Oral lozenges of heat-killed probiotics and postbiotics}

Three probiotic strains known for their antipathogenic against oral pathogens, namely $L$. salivarius subsp. salicinius AP-32, L. paracasei ET-66, and L. plantarum
LPL28, were obtained from Bioflag biotech. Co. Ltd (Tainan, Taiwan). L. salivarius subsp. salicinius AP-32 was isolated from healthy human intestine and deposited in Food Industry Research and Development Institute, Taiwan (ID: BCRC 910437) and in Wuhan university, China (ID: CCTCC-M2011127); $L$. paracasei ET-66 was isolated from healthy human breast milk and deposited in Food Industry Research and Development Institute, Taiwan (ID: BCRC 910753) and in China General Microbiological Culture Collection Center, Beijing, China (ID: CGMCC-13514). L. plantarum LPL28 was isolated from fermented food Mizo and deposited in Food Industry Research and Development Institute, Taiwan (ID: BCRC 910536) and in China General Microbiological Culture Collection Center, Beijing, China (ID: CGMCC-17954).

We collected postbiotics from these three strains $(50 \mathrm{mg}$ of mixed postbiotics $/ 1 \mathrm{~g}$ of lozenge) to develop oral lozenges [46]. The detailed procedure for producing postbiotic powder is described as follow: Incubating three probiotic strains AP-32, ET-66 and LPL28 $\left(2 \times 10^{11}\right.$ colony-formation units [CFUs] $/ \mathrm{g}$ ) in De Man, Rogosa and Sharpe (MRS) media (Difco. Laboratories, Detroit, MI, USA) at $37^{\circ} \mathrm{C}$ for $48 \mathrm{~h}$ to obtain viable probiotics strains. Fermenting mixed probiotics stains of AP-32, ET-66 and LPL28 (the concentration of each probiotic strain was $1 \times$ $10^{9} \mathrm{CFU} / \mathrm{mL}$ ) with nitrogen sources (skimmed milk and soy bean) and carbohydrate sources (glucose, fructose) at $37^{\circ} \mathrm{C}$ for 16 hours. Collecting fermented supernatant (postbiotic solution) after centrifugation at $15,000 \mathrm{x}$. Pasteurizing fermented product with ultra-hightemperature (UHT) to $135-140^{\circ} \mathrm{C}$ for 4 seconds. Then spray-drying fermented solution into postbiotic powder. The major nutritional components of postbiotic powder (per $100 \mathrm{~g}$ contribution) were crude protein $15.9 \mathrm{~g}$, crude fat $1.9 \mathrm{~g}$, saturated fat $0.21 \mathrm{~g}$, carbohydrate $65.3 \mathrm{~g}$, sugar $3.799 \mathrm{~g}$, glucose $0.553 \mathrm{~g}$, sucrose $0.082 \mathrm{~g}$, maltose $0.667 \mathrm{~g}$, lactose $2.497 \mathrm{~g}$, sodium $3062.7 \mathrm{mg}$, and calories 341.9 Kcal.

We incubated two probiotic strains $L$. salivarius subsp. salicinius $\mathrm{AP}-32$ and $L$. paracasei ET-66 $\left(2 \times 10^{11}\right.$ colony-formation units [CFUs]/g) in De Man, Rogosa and Sharpe (MRS) media (Difco. Laboratories, Detroit, MI, USA) at $37^{\circ} \mathrm{C}$ for $48 \mathrm{~h}$ to obtain viable probiotics strains [22]. Fermentation and centrifugation procedure was the same as making postbiotic product. Collecting and pasteurizing pellet with ultra-high-temperature (UHT) to $135-140^{\circ} \mathrm{C}$ for 4 seconds. Freeze-drying the pasteurized pellet for $40 \mathrm{hr}$, and then obtaining heatkilled probiotic powder. The heat-killed probiotic oral lozenges were composed of $10^{10} \mathrm{CFUs} / \mathrm{g}$ of cells. Furthermore, food-grade D-sorbitol, erythritol, fructooligosaccharides, lactose, magnesium stearate, silica, and sucralose were used to prepare placebo oral 
lozenges. Furthermore, food-grade D-sorbitol, erythritol, fructooligosaccharides, lactose, magnesium stearate, silica, and sucralose were used to prepare placebo oral lozenges.

\section{Oral pathogenic bacteria}

We used tryptic soy broth (TSB; Merck KGaA, Darmstadt, Germany) supplemented with 5\% sheep's blood to cultivate $P$. gingivalis and $F$. nucleatum subsp. polymorphum and brain heart infusion (BHI; Merck $\mathrm{KGaA}$, Darmstadt, Germany) broth for culturing $A$. actinomycetemcomitans. Additionally, TSB was used to cultivate Streptococcus mutans. We incubated pathogens at $37^{\circ} \mathrm{C}(48 \mathrm{~h})$ for subsequent antibacterial tests. S. mutans BCRC 10793T, P. gingivalis BCRC 17689, P. gingivalis BCRC 17688, $F$. nucleatum subsp. polymorphum BCRC 17679, and $A$. actinomycetemcomitans BCRC 14405 were obtained from Bioresource Collection and Research Center (BCRC), Hsinchu, Taiwan.

\section{Analyzing bacteriostatic activities}

The three probiotic strains were individually cultured in MRS media at $37^{\circ} \mathrm{C}$ for $20 \mathrm{~h}$. Then, $4.9 \mathrm{~mL}$ of supernatants were collected and mixed with oral pathogenic bacteria $\left(10^{6} \mathrm{CFUs} / 0.1 \mathrm{~mL}\right)$ after which the mixed solution was incubated at $37^{\circ} \mathrm{C}$ for $48 \mathrm{~h}$. Subsequently, the CFUs of pathogenic bacteria in each tube were calculated. Furthermore, the CFUs of oral pathogens were compared with the control media, which contained pathogens without postbiotic treatment.

The bacteriostatic activities of postbiotic and heat-killed probiotic lozenges were tested according to the same protocol. The experimental lozenges were dissolved in either a TSB or BHI medium at $0.1 \mathrm{~g} / \mathrm{mL}$ concentration, and then, oral pathogens $\left(10^{6} \mathrm{CFU}\right)$ were introduced into the lozenge solutions and coincubated at $37^{\circ} \mathrm{C}$ for 2 (S. mutans) or 3 (P. gingivalis, F. nucleatum subsp. polymorphum, and A. actinomycetemcomitans) days. Furthermore, the CFUs of pathogenic bacteria in each tube were calculated. We measured the survival rates of the oral pathogens by using the following formula: $\mathrm{CFU}_{\text {experimental group }} / \mathrm{CFU}_{\text {control media }}(\%)$. The inhibition rates of the oral pathogens were determined using the following formula: $1-$ survival rate (\%). The metabolites of L. rhamnosus GG (LGG) purchased from Chr. Hansen, Hoersholm, Denmark were tested as positive control.

\section{Participants}

In total, 75 participants (on-smokers, free from systemic diseases) between 20 and 40 years of age and with $S$. mutans $>10^{5} \mathrm{CFUs} / \mathrm{mL}$ in their saliva samples were recruited. Their average age was $26.29 \pm 5.59$ years. The initial amount of $S$. mutans in their saliva (CFUs $/ \mathrm{mL}$ ) was $4.25 * 10^{6} \pm 2.90 * 10^{6}$, and their plaque weight was $0.37 \pm 0.16 \mathrm{~g}$. All clinical tests were performed according to the guidelines of the Ministry of Health and Welfare, Taiwan (Health Food Evaluation No. 88037803). All participants were randomly and blindly assigned to three groups: placebo, heat-killed probiotics, and postbiotics (25 participants in each group). Participants were asked to clean their oral cavity and then consume three oral lozenges ( $3 \mathrm{~g}$ ) every day for 4 weeks [47]. We collected and measured oral microbiota, IgA levels, and oral pathogens in $2-\mathrm{mL}$ saliva samples at weeks 0,2 , and 4 . Additionally, total plaque and oral health questionnaire were analyzed at weeks 0,2 , and 4 . The protocols for evaluating the uptake of postbiotic products, colleting human saliva samples, and administering subjective questionnaires were approved by the Institutional Review Board of Chung Shan Medical University, Taiwan (CS19052).

\section{Analysis of the populations of Lactobacillus, Bifidobacterium, $S$. mutans, and total aerobic bacteria in the oral cavity}

For analyzing the populations of Lactobacillus, Bifidobacterium, total aerobic bacteria, and S. mutans in the oral cavity, $100-\mu \mathrm{L}$ saliva samples were cultured on MRS with $0.05 \%$ cysteine agar, plate count agar (PCA; Merck KGaA, Darmstadt, Germany), and mitis salivarius-bacitracin (MSB Agar) (Merck KGaA, Darmstadt, Germany) in triplicate, and CFUs on each plate were calculated. The change rates of oral pathogen were determined using the following formula: $\left(\mathrm{CFUS}_{\text {week } 2 \text { or } 4}-\mathrm{CFUS}_{\text {week } 0}\right) / \mathrm{CFUS}_{\text {week } 0}(\%)$.

\section{Next-generation sequencing analysis of oral microbiota}

Changes in oral microbiota were measured using the next-generation sequencing (NGS) technique. Microbial DNA was extracted from the saliva samples and sent to Genomics Co. Ltd. for NGS analysis. In brief, commercial specific primers (Genomics Co. Ltd., Taiwan) were used to amplify the amplicon DNA segments (16S rRNA and 16S V3-V4) by using the polymerase chain reaction (PCR) technique (Phusion High-Fidelity PCR Master Mix, New England Biolabs, USA). The PCR products of 400-450 bp were purified using the Qiagen Gel Extraction kit (Qiagen, Germany). Then, the TruSeq DNA PCR-free sample preparation kit (Illumina, USA) was used to generate sequencing libraries with provided index codes. The Qubit 2.0 Fluorometer (Thermo Scientific, USA) and Agilent Bioanalyzer 2100 system (Agilent Technologies, Inc., 
USA) were applied to confirm the library quality. Finally, the Illumina HiSeq 2500 platform was used to sequence and analyze the DNA library. QIIME software, version 1.7.0, was used to analyze NGS raw data [48]. The sizes of each single taxon of the groups were further analyzed through linear regression plots and linear discriminant analysis effect size (LEfSe) analysis (https://huttenhower.sph.harvard.edu/galaxy/) and the analysis protocol was followed the instruction in https://twbattaglia.gitbooks.io/introduction-to-qiime/ content/lefse.html.

\section{Measuring IgA and plaque}

The human IgA enzyme-linked immunosorbent assay (ELISA) kit (Invitrogen, Lot: 218315-003) was used to measure IgA concentrations of saliva samples in triplicate. The IgA concentration was analyzed at an optical density of $450-570 \mathrm{~nm}$ by using the ELISA reader. Plaque in the mouth was collected using a swab. Then, the dehydrated plaque was weighed. The weight of plague $(\mathrm{g})=$ total weight of samples with sample tubes $(\mathrm{g})$ - sample tubes $(\mathrm{g})$.

\section{Questionnaire of dental problems and gastrointestinal symptoms}

Self-report questionnaires were used to evaluate common dental symptoms and a gastrointestinal symptom [23]. All participants completed the questionnaire at weeks 0,2 , and 4 after the intervention. Participants could give the following responses: $0=$ no symptom; 1 = mild; $2=$ medium; $3=$ serious.

\section{Statistics}

GraphPad Prism software (San Diego, CA, USA) was applied to perform statistical analysis of collected data. Data of bacterial colonies in oral and salivary $\operatorname{IgA}$ are presented as medians. Each test was performed triplicate. The rest of the data are presented as means \pm standard deviations (SDs) or means. Two-tailed t-tests were performed to analyze the statistical differences in experimental results. Statistical difference was indicated by $\mathrm{p}<0.05$. A significant statistical difference was observed in the treatment data of the experimental groups compared with their pretreatment data at week 0 $(\# \mathrm{p}<0.05)$ and compared with the placebo group data at week $4(* \mathrm{p}<0.05)$.

\section{AUTHOR CONTRIBUTIONS}

Conceptualization, Chiao-Wen Lin, Yi-Tzu Chen, Shun-Fa Yang and Hsieh-Hsun Ho; Methodology, YiWei Kuo, Yao-Tsung Yeh, Ching-Wei Chen, Yu-Fen Huang, and Chen-Hung Hsu; Investigation: Wen-Yang
Lin; Formal analysis: Yi-Wei Kuo; Data acquisition and classification: Jia-Hung Lin, Chi-Huei Lin, Cheng-Ruei Liu and Jui-Fen Chen; Data Visualization, Wen-Yang Lin; Writing - original draft preparation, Wen-Yang Lin; Writing - review and editing: Chiao-Wen Lin, Hsieh-Hsun Ho, Yi-Wei Kuo, Wen-Yang Lin, Pei-Shan Hsieh and Shun-Fa Yang.

\section{CONFLICTS OF INTEREST}

The authors declare that they have no conflicts of interest.

\section{FUNDING}

Bioflag Biotech Co., Ltd provided support in the form of salaries for authors, but did not have any additional role in the study design, data collection and analysis, decision to publish, or preparation of the manuscript.

\section{REFERENCES}

1. Wu RQ, Zhang DF, Tu E, Chen QM, Chen W. The mucosal immune system in the oral cavity-an orchestra of T cell diversity. Int J Oral Sci. 2014; 6:125-32. https://doi.org/10.1038/ijos.2014.48 PMID:25105816

2. McLean JS. Advancements toward a systems level understanding of the human oral microbiome. Front Cell Infect Microbiol. 2014; 4:98. https://doi.org/10.3389/fcimb.2014.00098 PMID:25120956

3. Perera M, Al-Hebshi NN, Speicher DJ, Perera I, Johnson NW. Emerging role of bacteria in oral carcinogenesis: a review with special reference to perio-pathogenic bacteria. J Oral Microbiol. 2016; 8:32762.

https://doi.org/10.3402/jom.v8.32762 PMID:27677454

4. Sharma N, Bhatia S, Sodhi AS, Batra N. Oral microbiome and health. AIMS Microbiol. 2018; 4:42-66. https://doi.org/10.3934/microbiol.2018.1.42 PMID:31294203

5. Jia G, Zhi A, Lai PF, Wang G, Xia Y, Xiong Z, Zhang H, Che N, Ai L. The oral microbiota - a mechanistic role for systemic diseases. Br Dent J. 2018; 224:447-55. https://doi.org/10.1038/sj.bdj.2018.217 PMID:29569607

6. Kilian M, Chapple IL, Hannig M, Marsh PD, Meuric V, Pedersen AM, Tonetti MS, Wade WG, Zaura E. The oral microbiome - an update for oral healthcare professionals. Br Dent J. 2016; 221:657-66.

https://doi.org/10.1038/sj.bdj.2016.865 PMID:27857087 
7. Dewhirst FE, Chen T, Izard J, Paster BJ, Tanner AC, Yu WH, Lakshmanan A, Wade WG. The human oral microbiome. J Bacteriol. 2010; 192:5002-17. https://doi.org/10.1128/JB.00542-10 PMID:20656903

8. Nath SG, Raveendran R. Microbial dysbiosis in periodontitis. J Indian Soc Periodontol. 2013; 17:543-5. https://doi.org/10.4103/0972-124X.118334 PMID:24174742

9. Jepsen K, Jepsen S. Antibiotics/antimicrobials: systemic and local administration in the therapy of mild to moderately advanced periodontitis. Periodontol 2000. 2016; 71:82-112. https://doi.org/10.1111/prd.12121 PMID:27045432

10. Bolstad Al, Jensen HB, Bakken V. Taxonomy, biology, and periodontal aspects of Fusobacterium nucleatum. Clin Microbiol Rev. 1996; 9:55-71.

https://doi.org/10.1128/CMR.9.1.55

PMID: 8665477

11. Nazir MA. Prevalence of periodontal disease, its association with systemic diseases and prevention. Int J Health Sci (Qassim). 2017; 11:72-80.

PMID:28539867

12. Blaizot A, Vergnes JN, Nuwwareh S, Amar J, Sixou M. Periodontal diseases and cardiovascular events: metaanalysis of observational studies. Int Dent J. 2009; 59:197-209.

PMID:19774803

13. Petersen PE, Ogawa $\mathrm{H}$. Strengthening the prevention of periodontal disease: the WHO approach. J Periodontol. 2005; 76:2187-93. https://doi.org/10.1902/jop.2005.76.12.2187 PMID:16332229

14. Morales A, Gandolfo A, Bravo J, Carvajal P, Silva N, Godoy C, Garcia-Sesnich J, Hoare A, Diaz P, Gamonal J. Microbiological and clinical effects of probiotics and antibiotics on nonsurgical treatment of chronic periodontitis: a randomized placebo- controlled trial with 9-month follow-up. J Appl Oral Sci. 2018; 26:e20170075.

https://doi.org/10.1590/1678-7757-2017-0075 PMID:29364340

15. de Vos P, Faas MM, Spasojevic M, Sikkema J. Encapsulation for preservation of functionality and targeted delivery of bioactive food components. Int Dairy J. 2010; 20:292-302.

https://doi.org/10.1016/j.idairyj.2009.11.008

16. Yamada M, Takahashi N, Matsuda Y, Sato K, Yokoji M, Sulijaya B, Maekawa T, Ushiki T, Mikami Y, Hayatsu M, Mizutani Y, Kishino S, Ogawa J, et al. A bacterial metabolite ameliorates periodontal pathogen-induced gingival epithelial barrier disruption via GPR40 signaling. Sci Rep. 2018; 8:9008. https://doi.org/10.1038/s41598-018-27408-y PMID:29899364

17. Salminen S, Collado MC, Endo A, Hill C, Lebeer S, Quigley EM, Sanders ME, Shamir R, Swann JR, Szajewska H, Vinderola G. The International Scientific Association of Probiotics and Prebiotics (ISAPP) consensus statement on the definition and scope of postbiotics. Nat Rev Gastroenterol Hepatol. 2021; 18:649-67.

https://doi.org/10.1038/s41575-021-00440-6 PMID:33948025

18. Wegh CA, Geerlings SY, Knol J, Roeselers G, Belzer C. Postbiotics and Their Potential Applications in Early Life Nutrition and Beyond. Int J Mol Sci. 2019; 20:4673.

https://doi.org/10.3390/ijms20194673 PMID:31547172

19. Ryan PM, Ross RP, Fitzgerald GF, Caplice NM, Stanton C. Sugar-coated: exopolysaccharide producing lactic acid bacteria for food and human health applications. Food Funct. 2015; 6:679-93. https://doi.org/10.1039/C4FO00529E PMID:25580594

20. Rad AH, Abbasi A, Kafil HS, Ganbarov K. Potential Pharmaceutical and Food Applications of Postbiotics: A Review. Curr Pharm Biotechnol. 2020; 21:1576-87. https://doi.org/10.2174/1389201021666200516154 833 PMID:32416671

21. Ishikawa KH, Bueno MR, Kawamoto D, Simionato MR, Mayer MP. Lactobacilli postbiotics reduce biofilm formation and alter transcription of virulence genes of Aggregatibacter actinomycetemcomitans. Mol Oral Microbiol. 2021; 36:92-102. https://doi.org/10.1111/omi.12330 PMID:33372378

22. Chen YT, Hsieh PS, Ho HH, Hsieh SH, Kuo YW, Yang SF, Lin CW. Antibacterial activity of viable and heat-killed probiotic strains against oral pathogens. Lett Appl Microbiol. 2020; 70:310-17. https://doi.org/10.1111/lam.13275 PMID:31955445

23. Lin CW, Chen YT, Ho HH, Hsieh PS, Kuo YW, Lin JH, Liu CR, Huang YF, Chen CW, Hsu CH, Lin WY, Yang SF. Lozenges with probiotic strains enhance oral immune response and health. Oral Dis. 2021. [Epub ahead of print].

https://doi.org/10.1111/odi.13854 PMID:33749084

24. Atlas RM. Handbook of microbiological media. CRC press. 2004. https://doi.org/10.1201/9781420039726

25. Bubnov RV, Babenko LP, Lazarenko LM, Mokrozub VV, Spivak MY. Specific properties of probiotic strains: relevance and benefits for the host. EPMA J. 2018; 9:205-23. https://doi.org/10.1007/s13167-018-0132-z PMID:29896319 
26. Burton JP, Chilcott CN, Moore CJ, Speiser G, Tagg JR. A preliminary study of the effect of probiotic Streptococcus salivarius $\mathrm{K} 12$ on oral malodour parameters. J Appl Microbiol. 2006; 100:754-64.

https://doi.org/10.1111/i.1365-2672.2006.02837.x PMID:16553730

27. Dani S, Prabhu A, Chaitra KR, Desai NC, Patil SR, Rajeev R. Assessment of Streptococcus mutans in healthy versus gingivitis and chronic periodontitis: A clinicomicrobiological study. Contemp Clin Dent. 2016; 7:529-34.

https://doi.org/10.4103/0976-237X.194114 PMID:27994423

28. Nakano K, Nomura R, Ooshima T. Streptococcus mutans and cardiovascular diseases. Jpn Dent Sci Rev. 2008; 44:29-37.

https://doi.org/10.1016/j.jdsr.2007.09.001

29. Pabst $O$. New concepts in the generation and functions of IgA. Nat Rev Immunol. 2012; 12:821-32. https://doi.org/10.1038/nri3322 PMID:23103985

30. Bishop NC, Blannin AK, Armstrong E, Rickman M, Gleeson M. Carbohydrate and fluid intake affect the saliva flow rate and IgA response to cycling. Med Sci Sports Exerc. 2000; 32:2046-51. https://doi.org/10.1097/00005768-200012000-00013 PMID:11128850

31. Chao YX, Rötzschke O, Tan EK. The role of IgA in COVID19. Brain Behav Immun. 2020; 87:182-3. https://doi.org/10.1016/i.bbi.2020.05.057 PMID:32454136

32. Nishihara T, Suzuki N, Yoneda M, Hirofuji T. Effects of Lactobacillus salivarius-containing tablets on caries risk factors: a randomized open-label clinical trial. BMC Oral Health. 2014; 14:110.

https://doi.org/10.1186/1472-6831-14-110 PMID:25178882

33. Krzyściak W, Kościelniak D, Papież M, Vyhouskaya $P$, Zagórska-Świeży K, Kołodziej I, Bystrowska B, Jurczak A. Effect of a Lactobacillus Salivarius Probiotic on a Double-Species Streptococcus Mutans and Candida Albicans Caries Biofilm. Nutrients. 2017; 9:1242.

https://doi.org/10.3390/nu9111242

PMID:29135948

34. Hsieh PS, Tsai YC, Chen YC, Teh SF, Ou CM, King VA. Eradication of Helicobacter pylori infection by the probiotic strains Lactobacillus johnsonii $\mathrm{MH}-68$ and $\mathrm{L}$. salivarius ssp. salicinius AP-32. Helicobacter. 2012; 17:466-77.

https://doi.org/10.1111/i.1523-5378.2012.00992.x PMID:23067294

35. Lin WY, Kuo YW, Chen CW, Huang YF, Hsu CH, Lin JH, Liu CR, Chen JF, Hsia KC, Ho HH. Viable and Heat-Killed
Probiotic Strains Improve Oral Immunity by Elevating the IgA Concentration in the Oral Mucosa. Curr Microbiol. 2021; 78:3541-9.

https://doi.org/10.1007/s00284-021-02569-8

PMID:34345965

36. Hsieh PS, Ho HH, Tsao SP, Hsieh SH, Lin WY, Chen JF, Kuo YW, Tsai SY, Huang HY. Multi-strain probiotic supplement attenuates streptozotocin-induced type-2 diabetes by reducing inflammation and $\beta$-cell death in rats. PLoS One. 2021; 16:e0251646.

https://doi.org/10.1371/journal.pone.0251646 PMID:34166387

37. Guan X, Li W, Meng H. A double-edged sword: role of butyrate in the oral cavity and the gut. Mol Oral Microbiol. 2021; 36:121-31. https://doi.org/10.1111/omi.12322 PMID:33155411

38. Chang MC, Wang $\mathrm{TM}$, Chien $\mathrm{HH}$, Pan $\mathrm{YH}$, Tsai $\mathrm{YL}$, Jeng PY, Lin LD, Jeng JH. Effect of butyrate, a bacterial byproduct, on the viability and ICAM-1 expression/production of human vascular endothelial cells: role in infectious pulpal/periapical diseases. Int Endod J. 2022; 55:38-53.

https://doi.org/10.1111/iej.13614

PMID:34420220

39. Chang MC, Chen YJ, Lian YC, Chang BE, Huang CC, Huang $W L$, Pan $\mathrm{YH}$, Jeng JH. Butyrate Stimulates Histone H3 Acetylation, 8-Isoprostane Production, RANKL Expression, and Regulated Osteoprotegerin Expression/Secretion in MG-63 Osteoblastic Cells. Int J Mol Sci. 2018; 19:4071. https://doi.org/10.3390/ijms19124071 PMID:30562925

40. Singh TP, Natraj BH. Next-generation probiotics: a promising approach towards designing personalized medicine. Crit Rev Microbiol. 2021; 47:479-98. https://doi.org/10.1080/1040841X.2021.1902940 PMID:33822669

41. Hampelska K, Jaworska MM, Babalska ZŁ, Karpiński TM. The Role of Oral Microbiota in Intra-Oral Halitosis. J Clin Med. 2020; 9:2484. https://doi.org/10.3390/jcm9082484 PMID:32748883

42. Jiang S, Gao X, Jin L, Lo EC. Salivary Microbiome Diversity in Caries-Free and Caries-Affected Children. Int J Mol Sci. 2016; 17:1978.

https://doi.org/10.3390/ijms17121978 PMID:27898021

43. Nadkarni MA, Browne GV, Chhour KL, Byun R, Nguyen KA, Chapple CC, Jacques NA, Hunter N. Pattern of distribution of Prevotella species/phylotypes associated with healthy gingiva and periodontal disease. Eur J Clin Microbiol Infect Dis. 2012; 31:2989-99. 
https://doi.org/10.1007/s10096-012-1651-5

PMID:22684253

44. Gliga S, Devaux M, Gosset Woimant M, Mompoint D, Perronne C, Davido B. Actinomyces graevenitzii pulmonary abscess mimicking tuberculosis in a healthy young man. Can Respir J. 2014; 21:e75-7. https://doi.org/10.1155/2014/841480 PMID:25493591

45. Fujita $\mathrm{Y}$, likura $\mathrm{M}$, Horio $\mathrm{Y}$, Ohkusu K, Kobayashi $\mathrm{N}$. Pulmonary Actinomyces graevenitzii infection presenting as organizing pneumonia diagnosed by PCR analysis. J Med Microbiol. 2012; 61:1156-8. https://doi.org/10.1099/imm.0.040394-0 PMID:22516132

46. Stašková A, Sondorová $M$, Nemcová $R$, Kačírová J, Mad'ar M. Antimicrobial and Antibiofilm Activity of the Probiotic Strain Streptococcus salivarius K12 against Oral Potential Pathogens. Antibiotics (Basel). 2021; 10:793. https://doi.org/10.3390/antibiotics10070793

PMID: $\underline{34209988}$

47. Sutula J, Coulthwaite LA, Thomas LV, Verran J. The effect of a commercial probiotic drink containing Lactobacillus casei strain Shirota on oral health in healthy dentate people. Microb Ecol Health Dis. 2013; 24.

https://doi.org/10.3402/mehd.v24i0.21003

PMID:24179468

48. Caporaso JG, Kuczynski J, Stombaugh J, Bittinger K, Bushman FD, Costello EK, Fierer N, Peña AG, Goodrich JK, Gordon JI, Huttley GA, Kelley ST, Knights D, et al. QIIME allows analysis of high-throughput community sequencing data. Nat Methods. 2010; 7:335-6.

https://doi.org/10.1038/nmeth.f.303

PMID:20383131 


\section{SUPPLEMENTARY MATERIALS}

\section{Supplementary Figures}

\section{Section I.}

\section{In vitro screening of anti-bacterial activity}

Selecting postbiotics derived from L. salivarius subsp. salicinius AP-32, L. paracasei ET-66, and L. plantarum LPL-28 by anti-oral bacterial test (S. mutans, P. gingivalis,

F. nucleatum subsp. polymorphum, and A. actinomycetemcomitans)

\section{Section II.}

\section{Clinical Trial}

Participants were asked to clean their oral cavity and then consume three oral lozenges $(3 \mathrm{~g})$ every day for 4 weeks. We collected and measured oral microbiota, IgA levels, and oral pathogens in 2-mL saliva samples at weeks 0,2 , and 4

\section{Week 0}

Salivary sample collection/ NGS analysis

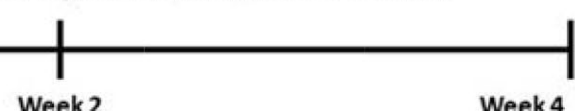

Week 2

Salivary sample collection

Week 4
Salivary sample collection/ NGS analysis

(mixed postbiotics of AP-32, ET-66, and LPL-28)

2. Heat-killed probiotic oral lozenges group (mixed heatkilled AP-32 and ET-66)

3. Placebogroup $(n=25)$

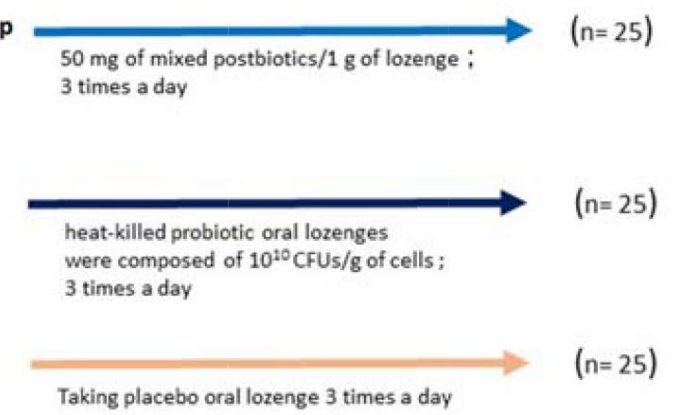

Supplementary Figure 1. Experimental design.

\section{Plaque in oral}

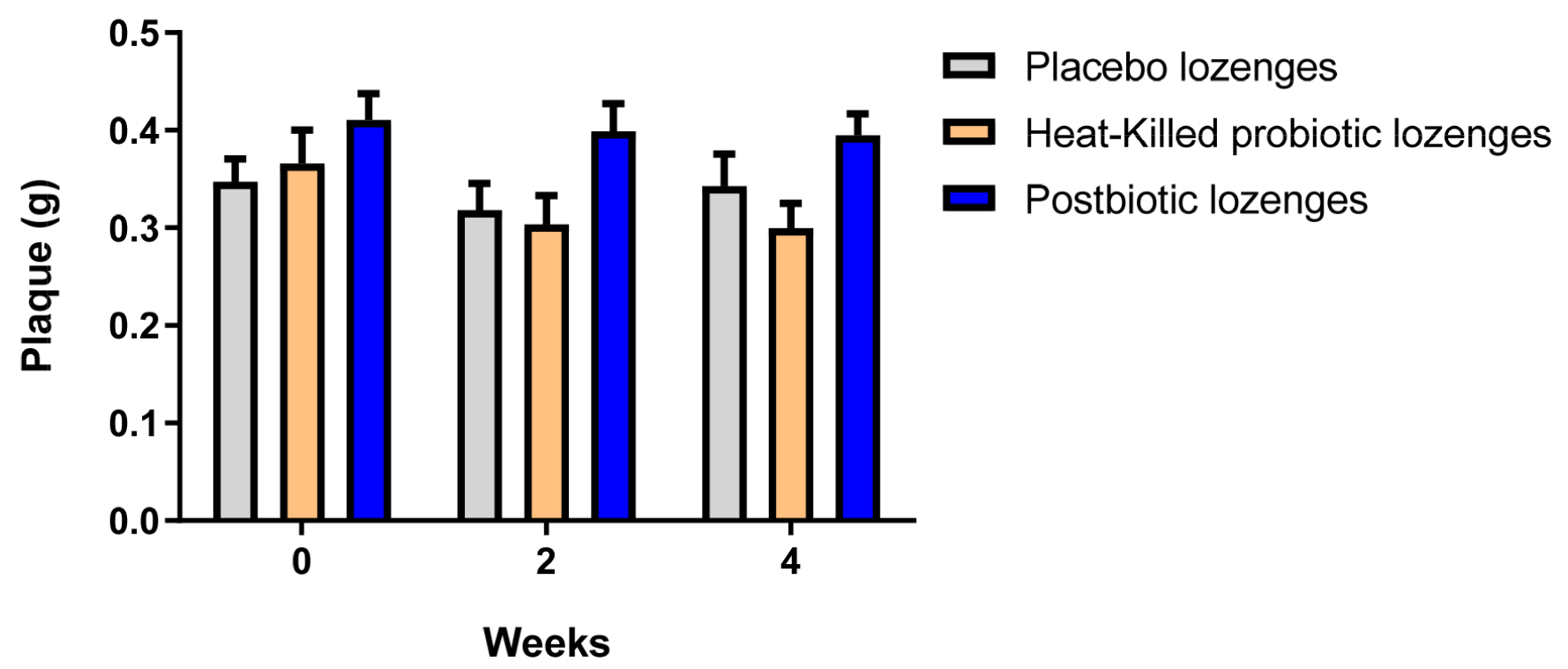

Supplementary Figure 2. Plaque weight analyses. The oral lozenges contained postbiotics or heat-killed cells. Participants in the control group consumed placebo lozenges without the postbiotic content (no significant difference was observed between the groups). Data are presented as mean \pm SD. 


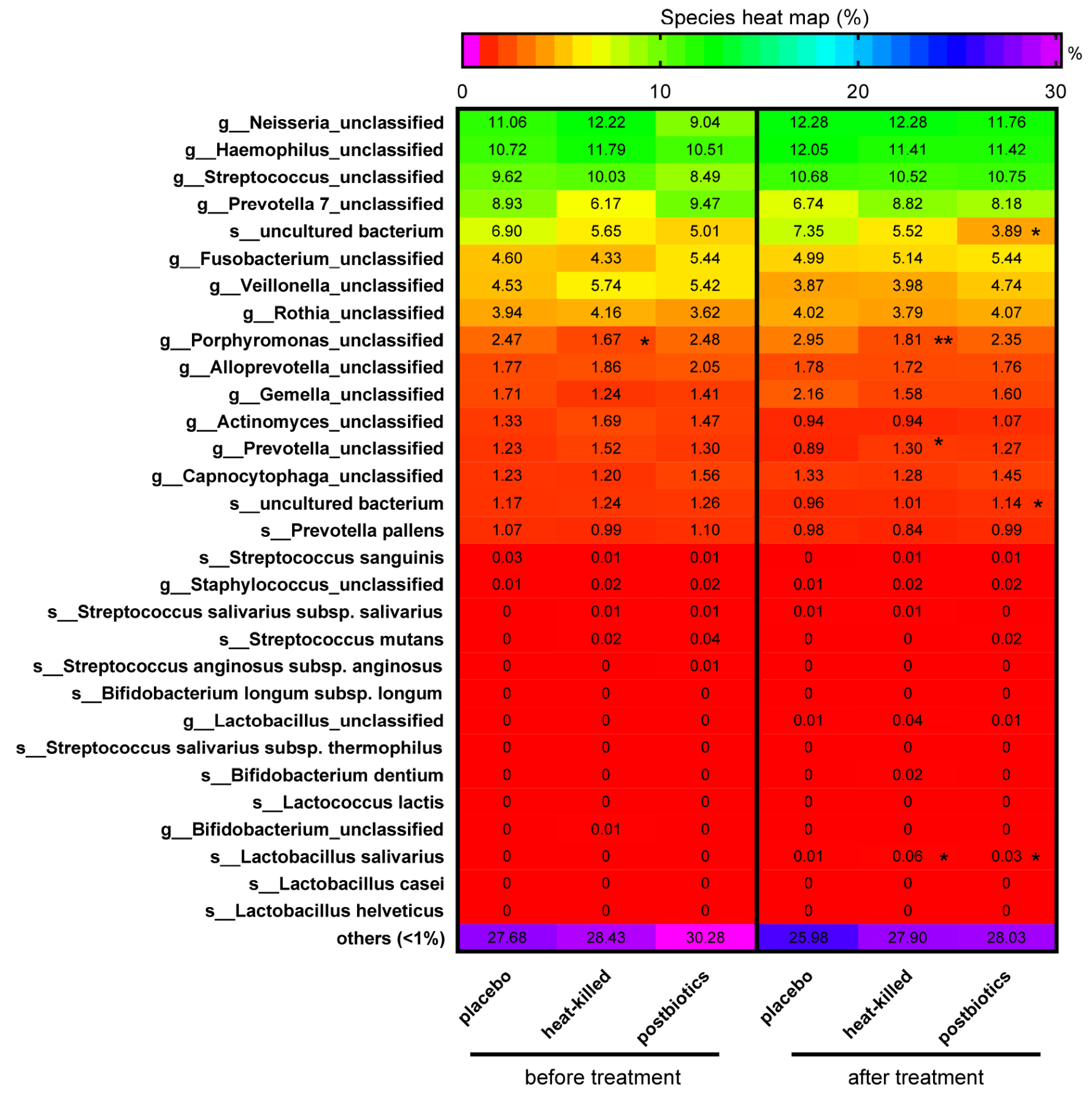

Supplementary Figure 3. NGS for detecting changes in oral microbiota with the intervention of oral lozenges. Heatmap presents oral microbiota changes (genus, \%) in participants' saliva before and after 4 weeks of consuming oral lozenge. Participants in the control group consumed placebo lozenges without the postbiotic content $\left({ }^{*} p<0.05\right.$ and ${ }^{* *} p<0.01$ indicate significant change compared with the placebo group). Data are presented as means. 


\section{Supplementary Tables}

Supplementary Table 1. Analyses of the oral health questionnaire.

\begin{tabular}{lccccccccc}
\hline & \multirow{2}{*}{ 0W } & & \multicolumn{2}{c}{ Placebo } & \multicolumn{3}{c}{ Heat-killed probiotics } & \multicolumn{3}{c}{ Postbiotics } \\
\cline { 3 - 10 } & & $\mathbf{2 W}$ & $\mathbf{4 W}$ & $\mathbf{0 W}$ & $\mathbf{2 W}$ & $\mathbf{4 W}$ & $\mathbf{0 W}$ & $\mathbf{2 W}$ & $\mathbf{4 W}$ \\
\hline $\begin{array}{l}\text { Toothache / } \\
\text { Gingival Swelling }\end{array}$ & $0.4 \pm 0.71$ & $0.2 \pm 0.41$ & $0.16 \pm 0.47$ & $0.31 \pm 0.68$ & $0.19 \pm 0.49$ & $0.19 \pm 0.69$ & $0.48 \pm 0.59$ & $0.28 \pm 0.54$ & $0.16 \pm 0.37$ \\
$\begin{array}{l}\text { Teeth bleeding, } \\
\text { while brushing }\end{array}$ & $0.48 \pm 0.78$ & $0.44 \pm 0.65$ & $0.44 \pm 0.65$ & $0.46 \pm 0.81$ & $0.27 \pm 0.53$ & $0.31 \pm 0.62$ & $0.44 \pm 0.58$ & $0.28 \pm 0.61$ & $0.16 \pm 0.37$ \\
$\begin{array}{l}\text { Ruptured mouth or } \\
\text { pustule formation }\end{array}$ & $0.6 \pm 0.76$ & $0.72 \pm 0.98$ & $0.52 \pm 0.82$ & $0.5 \pm 0.71$ & $0.23 \pm 0.51^{\mathrm{a}}$ & $0.15 \pm 0.37^{\mathrm{a}}$ & $0.44 \pm 0.82$ & $0.04 \pm 0.2^{\mathrm{b}}$ & $0.08 \pm 0.28^{\mathrm{a}}$ \\
$\begin{array}{l}\text { Sore throat } \\
\text { Drool }^{\mathrm{c}}\end{array}$ & $0.48 \pm 0.59$ & $0.4 \pm 0.87$ & $0.36 \pm 0.76$ & $0.42 \pm 0.7$ & $0.19 \pm 0.49$ & $0.19 \pm 0.4$ & $0.28 \pm 0.46$ & $0.2 \pm 0.41$ & $0.16 \pm 0.47$ \\
Cough $^{\mathrm{c}}$ & $0.32 \pm 0.56$ & $0.52 \pm 0.82$ & $0.48 \pm 0.65$ & $0.38 \pm 0.57$ & $0.12 \pm 0.33^{\mathrm{a}}$ & $0.15 \pm 0.46^{\mathrm{a}}$ & $0.6 \pm 0.76$ & $0.12 \pm 0.33^{\mathrm{a}}$ & $0.16 \pm 0.37^{\mathrm{a}}$ \\
\hline
\end{tabular}

aSignificant difference compared with the placebo group, $\mathrm{p}<0.05$.

${ }^{b}$ Highly significant difference compared with the placebo group, $\mathrm{p}<0.01$.

'Scores were evaluated according to severity: 3 points = severe; 2 points = normal; 1 point = mild; 0 point = none; the data are presented as mean \pm SD collected from all groups.

Supplementary Table 2. Intestinal symptoms based on health survey evaluationc.

\begin{tabular}{lccccccccc}
\hline & \multirow{2}{*}{ 0W } & & \multicolumn{2}{c}{ Placebo } & \multicolumn{3}{c}{ Heat-killed probiotics } & \multicolumn{3}{c}{ Postbiotics } \\
\cline { 3 - 10 } & & $\mathbf{2 W}$ & $\mathbf{4 W}$ & $\mathbf{0 W}$ & $\mathbf{2 W}$ & $\mathbf{4 W}$ & $\mathbf{0 W}$ & $\mathbf{2 W}$ & $\mathbf{4 W}$ \\
\hline Defecation $^{\mathrm{d}}$ & $0.08 \pm 0.28$ & $0.2 \pm 0.41$ & $0.36 \pm 0.49$ & $0.23 \pm 0.43$ & $0.38 \pm 0.5$ & $0.46 \pm 0.58$ & $0.2 \pm 0.41$ & $0.4 \pm 0.5$ & $0.32 \pm 0.48$ \\
Constipation $^{\mathrm{c}}$ & $0.48 \pm 0.65$ & $0.44 \pm 0.65$ & $0.6 \pm 0.71$ & $0.31 \pm 0.68$ & $0.15 \pm 0.46$ & $0.15 \pm 0.46^{\mathrm{a}}$ & $0.28 \pm 0.46$ & $0.16 \pm 0.37$ & $0.12 \pm 0.33^{\mathrm{b}}$ \\
Diarrhea $^{\mathrm{c}}$ & $0.2 \pm 0.41$ & $0.32 \pm 0.63$ & $0.36 \pm 0.64$ & $0.27 \pm 0.53$ & $0.15 \pm 0.46$ & $0.15 \pm 0.46$ & $0.12 \pm 0.33$ & $0.24 \pm 0.66$ & $0.16 \pm 0.37$ \\
Stomach pain $^{\mathrm{c}}$ & $0.68 \pm 0.8$ & $0.4 \pm 0.65$ & $0.32 \pm 0.63$ & $0.35 \pm 0.49$ & $0.23 \pm 0.43$ & $0.23 \pm 0.43$ & $0.36 \pm 0.49$ & $0.12 \pm 0.33$ & $0.16 \pm 0.37$ \\
Gastroesophageal $^{\mathrm{c}}$ & $0.68 \pm 0.9$ & $0.48 \pm 0.65$ & $0.52 \pm 0.71$ & $0.31 \pm 0.47$ & $0.15 \pm 0.37^{\mathrm{a}}$ & $0.19 \pm 0.4^{\mathrm{a}}$ & $0.28 \pm 0.46$ & $0.08 \pm 0.28^{\mathrm{b}}$ & $0.12 \pm 0.33^{\mathrm{a}}$ \\
Reflux $^{\mathrm{c}}$ & & & & & & & & & \\
Cold $^{\mathrm{c}}$ & $0.36 \pm 0.57$ & $0.44 \pm 0.71$ & $0.56 \pm 0.82$ & $0.46 \pm 0.58$ & $0.23 \pm 0.51$ & $0.12 \pm 0.43^{\mathrm{a}}$ & $0.32 \pm 0.47$ & $0.2 \pm 0.41$ & $0.16 \pm 0.37^{\mathrm{a}}$ \\
Drowsiness $^{\mathrm{c}}$ & $0.36 \pm 0.7$ & $0.4 \pm 0.58$ & $0.52 \pm 0.65$ & $0.31 \pm 0.47$ & $0.15 \pm 0.37$ & $0.08 \pm 0.27^{\mathrm{b}}$ & $0.32 \pm 0.56$ & $0.16 \pm 0.37$ & $0.12 \pm 0.33^{\mathrm{b}}$ \\
\hline
\end{tabular}

aSignificant difference compared with the placebo group, $\mathrm{p}<0.05$.

${ }^{b}$ Highly significant difference compared with the placebo group, $p<0.01$.

'Scores were evaluated according to severity: 3 points = severe; 2 points = normal; 1 point = mild; 0 point = none; the data are presented as mean \pm SD collected from all groups.

${ }^{\mathrm{d}}$ Scores were analyzed based on frequency: $>3$ days $=2$ points; once every 3 days $=1$ point; more than once a day $=0$ points. 